\title{
Presentations for vertex-transitive graphs
}

\author{
Agelos Georgakopoulos ${ }^{1}\left[\right.$ [ $\cdot$ Alex Wendland ${ }^{1}$
}

Received: 27 August 2020 / Accepted: 14 August 2021 / Published online: 4 October 2021

(c) The Author(s) 2021

\section{Abstract}

We generalise the standard constructions of a Cayley graph in terms of a group presentation by allowing some vertices to obey different relators than others. The resulting notion of presentation allows us to represent every vertex-transitive graph.

Keywords Group presentation · Cayley graph · Bi-Cayley graph · Vertex-transitive

\section{Introduction}

Every Cayley graph is vertex-transitive but the converse is not true, with the Petersen graph being a well-known example. A lot of research focuses on understanding how much larger the class of vertex-transitive graphs is or, what is essentially the same, on extending results from Cayley graphs to vertex-transitive graphs, see e.g. $[4,7,17$, $19,20,25]$ and references therein. This paper offers a new algebraic way of defining graphs, which we will prove to have the power to present all vertex-transitive graphs.

The idea is to still define our graphs by means of generators and relators similarly to Cayley graphs defined via group presentations, but we now allow different vertices to obey different sets of relators. The fewer 'types' of vertices we have the closer our graph is to being a Cayley graph. This is perhaps best explained with an example: in Fig. 1 we have directed and labelled the Petersen graph with two letters $r$ and $b$ that make it look almost like a Cayley graph. But a closer look shows that if we start at any exterior vertex $v$ and follow a sequence of edges labelled brbrr then we return to $v$, while this is not true if $v$ is one of the interior vertices. In the latter case, $b r r b r$ is an example of a word that gives rise to a cycle.

This example motivates our definition of a partite presentation, which prescribes a number of types of vertices, and a set of relators for each type. Moreover, it entails

\footnotetext{
Agelos Georgakopoulos: Supported by the European Research Council (ERC) under the European Union's Horizon 2020 research and innovation programme (Grant Agreement No. 639046).

$凶$ Agelos Georgakopoulos

A.Georgakopoulos@warwick.ac.uk

1 Department of Mathematics, University of Warwick, Coventry CV4 7AL, UK
} 
Fig. 1 The Petersen graph, labelled by two letters $r$ (for red) and $b$ (for blue). The cycle obtained by reading the 'relation' rbrrb starting at the top square vertex is depicted in bold lines

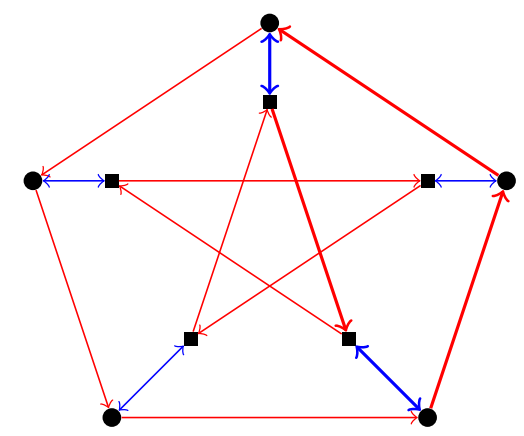

a set of generators, and for each generator $s$ it prescribes the type of end-vertex of an edge labelled $s$ for each type of starting vertex. The precise definition of partite presentations in the case where there are only two types of vertices, which we call 2partite presentations, is given in Sect. 3. The case with more classes is more involved, and it is given in Sect. 5.

We show how each partite presentation defines a graph, by imitating the standard definitions of a Cayley graph via a group presentation: either as a quotient of a free group by the normal subgroup generated by the relators (Definition 3.2), or as the 1-skeleton of the universal cover of the presentation complex (Definition 3.7). The resulting partite Cayley graph is always regular, with vertex-degree determined by the generating set, and it admits a group of automorphisms acting on its vertices semiregularly and with as many orbits as the number of types of vertices prescribed by its presentation (Proposition 5.14). In particular, 2-partite presentations always give rise to bi-Cayley graphs. We prove this, as well as a converse statement, in Sect. 4.

Our main result says that our formalism of partite Cayley graphs is general enough to describe all vertex-transitive graph:

Theorem 1.1 Every countable, vertex-transitive, graph has a partite presentation.

In general, for the proof of this we allow for the vertex types to be in bijection with the vertex set of the graph in question. It would be interesting to study how much the number of vertex types can be reduced, see Sect. 7. As we remark there, there are vertextransitive graphs that require infinitely many vertex types in any partite presentation; most Diestel-Leader graphs [6] have this property. In the converse direction, we show, in Sect. 6, that every line graph of a Cayley graph $\Gamma$ admits a partite presentation with at most as many vertex types as the number of generators of $\Gamma$.

The proof of Theorem 1.1 involves decomposing the edge-set into cycles. This decomposition is not obvious, and it is related to a conjecture of Leighton [17] disproved by Marušič [20]; see Sect. 5.2 for more. 


\section{Preliminaries}

\subsection{Graphs and automorphisms}

We work with the notion of graph as defined by Gersten [9]. A graph $\Gamma$ comprises a set of vertices $V(\Gamma)$, and a set of directed edges $\vec{E}(\Gamma)$, endowed with a fix point free involution $^{-1}: \vec{E}(\Gamma) \rightarrow \vec{E}(\Gamma)$ and a terminus map $\tau: \vec{E}(\Gamma) \rightarrow V(\Gamma)$. Sometimes we will express the elements of $\vec{E}(\Gamma)$ as directed pairs $(v, w)$ with $v, w \in V(\Gamma)$, in which case we tacitly mean that $\tau((v, w))=w$ and $(v, w)^{-1}=(w, v)$.

A directed edge $e \in \vec{E}(\Gamma)$ is a loop, if $\tau(e)=\tau\left(e^{-1}\right)$. The degree $d(v)$ of a vertex $v \in V(\Gamma)$ is the cardinality of $\left|\tau^{-1}(v)\right|$. We say that $\Gamma$ is $k$-regular if $d(v)=k$ for every $v \in V(\Gamma)$.

To a graph $\Gamma$ we can associate the set of undirected edges, $E(\Gamma):=\vec{E}(\Gamma) /^{-1}$. Thus $|\vec{E}(\Gamma)|=2|E(\Gamma)|$ for every graph $\Gamma$.

Note that although we are talking about 'directed edges', we are not talking about 'directed graphs' in the sense of e.g. [5]. Our edges can be thought of as undirected pairs of vertices, but our formalism allows us to distinguish between two orientations for each of them. Moreover, our formalism allows for multiple edges between the same pair of vertices, and multiple loops at a single vertex. Thus the pair $(V(\Gamma), E(\Gamma))$ is a multigraph in the sense of [5].

A map of graphs $\phi: \Gamma \rightarrow \Delta$ is a pair of maps $\left(\phi_{V}: V(\Gamma) \rightarrow V(\Delta), \phi_{E}:\right.$ $\vec{E}(\Gamma) \rightarrow \vec{E}(\Delta))$ where $\phi_{E}$ commutes with ${ }^{-1}$ and $\phi_{V} \circ \tau=\tau \circ \phi_{E}$. For a graph $\Gamma$, an endomorphism is a map from $\Gamma$ to itself, and it is called an automorphism if $\phi_{V}$ and $\phi_{E}$ are bijections. The sets of these maps are denoted $\operatorname{End}(\Gamma)$ and $\operatorname{Aut}(\Gamma)$, respectively.

We say that $\Gamma$ is vertex-transitive if $\operatorname{Aut}(\Gamma)$ acts transitively on $V(\Gamma)$, and edgetransitive if $A u t(\Gamma)$ acts transitively on $E(\Gamma)$. We say that $\Gamma$ is arc-transitive, or symmetric, if $A u t(\Gamma)$ acts transitively on $\vec{E}(\Gamma)$. We say $\Gamma$ is semi-symmetric if it is edge-transitive and regular but not vertex-transitive.

Given a set of undirected edges $S \subset E(\Gamma)$ of a graph $\Gamma$ an orientation of $S$ is a subsets $O_{S} \subset \vec{E}(\Gamma)$ such that $O_{S} /^{-1}=S$, and $O_{S} \cap O_{S}^{-1}=\emptyset$.

A walk in $\Gamma$ is an alternating sequence $v_{0} e_{1} v_{1} \ldots e_{n} v_{n}$ of vertices and directed edges such that $\tau\left(e_{i}\right)=v_{i}$ and $\tau\left(e_{i}^{-1}\right)=v_{i-1}$ for every $1 \leq i \leq n$.

\subsection{Groups and Cayley graphs}

Given a group $G$ and a subset $S \subset G$, we define the (right) Cayley graph $\Gamma=$ $\operatorname{Cay}(G, S)$ to be the graph with vertex set $V(\Gamma)=G$ and directed edge set $\vec{E}(\Gamma)=$ $\{(g,(g s)) \mid g \in G, s \in S\}$. Unless otherwise stated, we are not assuming that $S$ generates $G$, so that Cayley graphs in this paper are not always connected. The group $G$ acts on $\Gamma$ by automorphisms, by multiplication (on vertices) on the left. 


\subsection{Colourings}

In this work a graph colouring will always refer to a colouring of the edges. A colouring of the undirected edges of $\Gamma$ is a map $c: E(\Gamma) \rightarrow X$ whereas a colouring of the directed edges is a map $c: \vec{E}(\Gamma) \rightarrow X$, where $X$ is an arbitrary set called the set of colours.

\subsection{Covering spaces}

A covering space (or cover) of a topological space $X$ is a topological space $C$ endowed with a continuous surjective map $\psi: C \rightarrow X$ such that for every $x \in X$, there exists an open neighbourhood $U$ of $x$, such that $\psi^{-1}(U)$ is a union of disjoint open sets in $C$, each of which is mapped homeomorphically onto $U$ by $\psi$.

Given a map of spaces $\phi: Y \rightarrow X$, and point $y \in Y$ such that $p(y)=x$, we obtain an induced map between fundamental groups $\phi_{*}: \pi_{1}(Y, y) \rightarrow \pi_{1}(X, x)$ by composition. For a covering map $\phi$ we know that $\phi_{*}$ is injective [13, Proposition 1.31]. If $C$ is arc-connected, and $\pi_{1}(C, c)=1$, i.e. $C$ is simply connected, we call $C$ the universal cover of $X$, which is well-known to be unique when it exists.

Given a cover $\psi: C \rightarrow X$ and a map $\phi: Y \rightarrow X$ (with $Y$ path connected and locally path connected) we obtain a lift $\tilde{\phi}: Y \rightarrow C$ (where $\phi=\psi \circ \tilde{\phi}$ ) of $\phi$ if and only if $\phi_{*}\left(\pi_{1}(Y, y)\right) \subset \psi_{*}\left(\pi_{1}(C, c)\right)$ [13, Proposition 1.33]. Moreover, for any preimage $c \in \psi^{-1}(x)$ we can choose $\tilde{\phi}(y)=c$.

Lastly we recall the classification of covering spaces:

Theorem 2.1 (Hatcher [13, Theorem 1.38]) Let X be a path-connected, locally pathconnected, and semilocally simply-connected topological space. Then there is a bijection between the set of isomorphism classes of path-connected covering spaces $\psi: C \rightarrow X$ and the set of subgroups (up to conjugation) of $\pi_{1}(X)$, obtained by associating the subgroup $\psi_{*}\left(\pi_{1}(C)\right)$ to the covering space $C$.

\section{2-partite presentations}

\subsection{Algebraic definition}

We start by recalling one of the standard definitions of a Cayley graph, in order to then adapt it into the definition of a 2-partite Cayley graph.

Let $G$ be a group. A presentation $\langle\mathcal{S} \mid \mathcal{R}\rangle$ of $G$ consists of a generating set $\mathcal{S} \subset G$ and a relator set $\mathcal{R} \subset F_{\mathcal{S}}$ such that $F_{\mathcal{S}} /\langle\langle\mathcal{R}\rangle\rangle=G$, where $F_{\mathcal{S}}$ denotes the free group with free generating set $\mathcal{S}$, and $\langle\langle\mathcal{R}\rangle\rangle$ denotes its normal subgroup generated by $\mathcal{R}$. For a group presentation $\langle\mathcal{S} \mid \mathcal{R}\rangle$, we can construct the Cayley graph $\operatorname{Cay}\langle\mathcal{S} \mid \mathcal{R}\rangle$ in the following manner. Let $T_{\mathcal{S}}$ be the $2|\mathcal{S}|$-regular tree defined by

$$
\begin{aligned}
& V\left(T_{\mathcal{S}}\right):=F_{\mathcal{S}}, \text { and } \\
& \vec{E}\left(T_{\mathcal{S}}\right):=\left\{(w, w s) \mid w \in F_{\mathcal{S}}, s \in \mathcal{S} \cup \mathcal{S}^{-1}\right\} .
\end{aligned}
$$


We endow $T_{\mathcal{S}}$ with a colouring $c: \vec{E}\left(T_{\mathcal{S}}\right) \rightarrow \mathcal{S} \cup \mathcal{S}^{-1}$ defined by $c(w, w s)=s$ and $c(w s, w)=s^{-1}$. Let $R:=\langle\langle\mathcal{R}\rangle\rangle$ be the normal closure of $\mathcal{R}$ in $F_{\mathcal{S}}$. Define an equivalence relation $\sim$ on $V\left(T_{\mathcal{S}}\right)=F_{\mathcal{S}}$ by letting $v \sim w$ whenever $v^{-1} w \in R$. Extend $\sim$ to $\vec{E}\left(T_{\mathcal{S}}\right)$ by demanding $e \sim d$ whenever $c(e)=c(d)$ and $\tau(e) \sim \tau(d)$ and $\tau\left(e^{-1}\right) \sim \tau\left(d^{-1}\right)$. Then $\operatorname{Cay}\langle\mathcal{S} \mid \mathcal{R}\rangle$ can be defined as the quotient $T_{\mathcal{S}} / \sim$. The corresponding covering map is denoted by $\eta: T_{\mathcal{S}} \rightarrow \operatorname{Cay}\langle\mathcal{S} \mid \mathcal{R}\rangle$. Note that as $\sim$ preserves $c$, we obtain a unique colouring $c^{\prime}: \vec{E}(\operatorname{Cay}\langle\mathcal{S} \mid \mathcal{R}\rangle) \rightarrow \mathcal{S} \cup \mathcal{S}^{-1}$ satisfying $c=c^{\prime} \circ \eta$.

This definition of the Cayley graph is standard. All Cayley graphs defined this way have even degrees: involutions in $\mathcal{S}$ give rise to pairs of 'parallel' edges with the same end-vertices. However, in certain contexts it is desirable to replace such pairs of parallel edges by single edges. To accommodate for this modification-which is important for us later as we want to capture odd-degree graphs such as the Petersen graph with our presentations - we now introduce modified presentations and Cayley graphs.

For a group presentation $\left\langle\mathcal{S} \mid \mathcal{R}^{\prime}\right\rangle$, we define the modified presentation $P=\langle\mathcal{U}, \mathcal{I} \mid \mathcal{R}\rangle$ where $\mathcal{I}:=\left\{s \in \mathcal{S}: s^{2} \in \mathcal{R}^{\prime}\right\}$ and $\mathcal{U}:=\mathcal{S} \backslash \mathcal{I}$. Define the corresponding modified free group $M F_{P}:=\left\langle\mathcal{S} \mid\left\{s^{2}: s \in \mathcal{I}\right\}\right\rangle$. (Thus $M F_{P}$ is a free product of infinite cyclic groups, one for each $s \in \mathcal{U}$, and cyclic groups of order 2, one for each $s \in \mathcal{I}$.) Let $\phi: F_{\mathcal{S}} \rightarrow M F_{P}$ be the unique homomorphism extending the identity on $F_{\mathcal{S}}$, as provided by the universal property of free groups, and let $\mathcal{R}:=\phi\left(\mathcal{R}^{\prime}\right) \backslash\{1\} \subset M F_{P}$. Define the $\left|\mathcal{S} \cup \mathcal{S}^{-1}\right|$-regular tree $T_{P}$ by

$$
\begin{aligned}
& V\left(T_{P}\right):=M F_{P} \\
& \vec{E}\left(T_{P}\right):=\left\{(w, w s) \mid w \in M F_{P}, s \in \mathcal{S} \cup \mathcal{S}^{-1}\right\} .
\end{aligned}
$$

We proceed as above to define the colouring $c$ and the relation $\sim$, and obtain the modified Cayley graph as the quotient $T_{P} / \sim$.

We now modify the above construction of the Cayley graph, to obtain our partite Cayley graphs. The basic idea is to partition the vertex set into two (and later more than two) classes $V_{1}, V_{2}$, obeying different sets of relators $\mathcal{R}_{0}, \mathcal{R}_{1}$. This bipartition creates the need to distinguish our generators too into two classes $\mathcal{S}_{1}, \mathcal{S}_{2}$, the former corresponding to edges staying in the same partition class, and the latter corresponding to edges incident with both classes $V_{1}, V_{2}$.

We will formally define a 2-partite presentation as a 4-tuple $P=\left\langle\mathcal{S}_{1}, \mathcal{S}_{2} \mid \mathcal{R}_{0}, \mathcal{R}_{1}\right\rangle$, and explain how this data is used to define a partite Cayley graph PCay $(P)$, in analogy with the above definition of a Cayley graph $\operatorname{Cay}\langle\mathcal{S} \mid \mathcal{R}\rangle$ corresponding to a group presentation $P=\langle\mathcal{S} \mid \mathcal{R}\rangle$. The set $\mathcal{S}_{1}$ is an arbitrary set of 'generators'. We partition $\mathcal{S}_{2}$ into two sets, $\mathcal{S}_{2}=\{\mathcal{U}, \mathcal{I}\}$, so that $\mathcal{S}_{1}, \mathcal{U}, \mathcal{I}$ are pairwise disjoint. Their union $\mathcal{S}:=\mathcal{S}_{1} \cup \mathcal{U} \cup \mathcal{I}$ will be our set of generators. The necessity of distinguishing $\mathcal{S}_{2}$ into $\mathcal{U}, \mathcal{I}$ is to allow for some involutions, namely the elements of $\mathcal{I}$, to give rise to single edges in our graphs, just like in the above definition of modified Cayley graph.

As in our definition of modified Cayley graph, we let $M F_{P}:=\left\langle\mathcal{S} \mid\left\{s^{2}: s \in \mathcal{I}\right\}\right\rangle$. Let $|\cdot|_{\mathcal{S}_{2}}$ be the unique homomorphism from $M F_{P}$ to $\mathbb{Z} / 2 \mathbb{Z}$ extending 


$$
|s|_{\mathcal{S}_{2}}=\left\{\begin{array}{ll}
0 & \text { if } s \in \mathcal{S}_{1} \\
1 & \text { if } s \in \mathcal{S}_{2}
\end{array} .\right.
$$

We have that $K:=\operatorname{Ker}\left(|\cdot|_{\mathcal{S}_{2}}\right)$ is an index-two subgroup of $M F_{P}$, and so its cosets $\tilde{V}_{1}:=K$ and $\tilde{V}_{2}:=\mathcal{S}_{2} K$ bipartition $M F_{P}$.

Definition 3.1 For any two sets $\mathcal{R}_{0}, \mathcal{R}_{1} \subset K$, called relator sets, we call the tuple $\left\langle\mathcal{S}_{1}, \mathcal{S}_{2} \mid \mathcal{R}_{0}, \mathcal{R}_{1}\right\rangle$ a 2-partite presentation.

(The restriction $\mathcal{R}_{i} \subset K$ does not have an analogue in the definition of Cayley graph; the intuition is that relators should start and finish at the same side of the bipartition $V_{1}, V_{2}$ because they are supposed to yield cycles in the graph.)

Given a 2-partite presentation $P=\left\langle\mathcal{S}_{1}, \mathcal{U}, \mathcal{I} \mid \mathcal{R}_{0}, \mathcal{R}_{1}\right\rangle$, recall that $M F_{P}=\langle\mathcal{S}|\left\{s^{2}\right.$ : $s \in \mathcal{I}\}\rangle$, and define the $\left(\left|\mathcal{S} \cup \mathcal{S}^{-1}\right|\right.$-regular $)$ tree $T_{P}$ by

$$
\begin{aligned}
& V\left(T_{P}\right):=M F_{P} \\
& \vec{E}\left(T_{P}\right):=\left\{(w, w s) \mid w \in M F_{P}, s \in \mathcal{S} \cup \mathcal{S}^{-1}\right\} .
\end{aligned}
$$

Define the subgroups

$R_{0}$ to be the normal closure of $\mathcal{R}_{0} \cup\left\{s r s^{-1}: r \in \mathcal{R}_{1}, s \in \mathcal{S}_{2}\right\}$ in $K$, and $R_{1}$ to be the normal closure of $\mathcal{R}_{1} \cup\left\{s r s^{-1}: r \in \mathcal{R}_{0}, s \in \mathcal{S}_{2}\right\}$ in $K$.

Here $R_{i} \leq K \leq M F_{P}$ is the analogue of the normal subgroup $R$ of $M F_{P}$ in the definition of Cay $(P)$, but now having two versions corresponding to our two classes of elements of $M F_{P}$, namely $\left\{\tilde{V}_{1}, \tilde{V}_{2}\right\}:=\left\{K, \mathcal{S}_{2} K\right\}$. In analogy with the relation $\sim$ above, we now write $v \sim w$ whenever $v^{-1} w \in R_{i}$ for $v, w \in \tilde{V}_{i}$. We extend $\sim$ to the edges of $T_{P}$ via $e \sim d$ if $c(e)=c(d), \tau(e) \sim \tau(d)$, and $\tau\left(e^{-1}\right) \sim \tau\left(d^{-1}\right)$.

Definition 3.2 The 2-partite Cayley graph $\operatorname{PCay}\left\langle\mathcal{S}_{1}, \mathcal{U}, \mathcal{I} \mid \mathcal{R}_{0}, \mathcal{R}_{1}\right\rangle=\operatorname{PCay}(P)=: \Gamma$ is the quotient $T_{P} / \sim$.

The edge set of $\Gamma$ can thus be written as $\vec{E}(\Gamma)=\vec{E}\left(T_{P}\right) / \sim$.

As before, we have a natural colouring $c: \vec{E}\left(T_{P}\right) \rightarrow \mathcal{S} \cup \mathcal{S}^{-1}$ defined by $c(w, w s)=s$, and as $\sim$ preserves $c$, the latter factors into $c^{\prime}: \vec{E}(\Gamma) \rightarrow \mathcal{S} \cup \mathcal{S}^{-1}$, i.e. the unique colouring satisfying $c=c^{\prime} \circ \eta$ where again $\eta$ denotes the projection map corresponding to $\sim$.

Note that this is a generalisation of the modified Cayley graph. When $\mathcal{I}=\emptyset$ we have a generalisation of the standard Cayley graph.

Borrowing terminology from groupoids, we define the vertex groups of our partite presentation to be $G_{i}:=K / R_{i}$ for $i \in \mathbb{Z} / 2 \mathbb{Z}$.

The condition $\mathcal{R}_{i} \subset K$ implies that if $v \sim w$ then $v$ and $w$ belong to the same coset $\tilde{V}_{0}$ or $\tilde{V}_{1}$ of $K$ in $M F_{P}$ by the definitions. Thus factoring by $\sim$ projects the bipartition $\left\{\tilde{V}_{0}, \tilde{V}_{1}\right\}$ of $M F_{P}$ into a bipartition $\left\{V_{0}, V_{1}\right\}$ of $V(\Gamma)$, with $V_{i}:=\tilde{V}_{i} / \sim$. It follows from these definitions that $G_{i}$ is in canonical bijection with $V_{i}$. 
As in the case of Cayley graphs, relators in the presentation yield closed walks in $\Gamma$, but now we need to start reading our relators at the correct side of the bipartition for this to be true: for every $i \in \mathbb{Z} / 2 \mathbb{Z}$ and each $r \in \mathcal{R}_{i}$ and $v \in V_{i}$, if we start at $v$ and follow the directed edges of $\Gamma$ with colours dictated by $r$ one-by-one, we finish our walk at $v$.

We now explain how the Petersen graph can be obtained as a 2-partite Cayley graph:

Example 3.3 Theorem 3.13 asserts that the Petersen graph $P(5,2)$ (Fig. 1) is isomorphic to $\operatorname{PCay}\left(\left\langle\mathcal{S}_{1}=\{a\}, \mathcal{U}=\emptyset, \mathcal{I}=\{b\} \mid \mathcal{R}_{0}=\left\{a^{5}, a b a^{2} b, b^{2}\right\}, \mathcal{R}_{1}=\left\{a^{5}\right\}\right\rangle\right)=$ PCay $\left\langle\{a\}, \emptyset,\{b\} \mid\left\{a^{5}, a b a^{2} b\right\},\left\{a^{5}\right\}\right\rangle$. For this presentation we have

- $M F_{P}=\left\langle a, b \mid b^{2}\right\rangle$, so that $T_{P}$ is the 3-regular tree;

- $K=\langle\langle a, b a b\rangle\rangle \leq M F_{P}$;

- $R_{0}=\left\langle\left\langle a^{5}, a b a^{2} b, b a^{5} b\right\rangle\right\rangle_{K}$, and

- $R_{1}=\left\langle\left\langle b a^{5} b, b a b a^{2}, a^{5}\right\rangle\right\rangle_{K}$.

(There are many alternative ways to present $\mathcal{R}_{0}, \mathcal{R}_{2}$ and $R_{1}=R_{2}$, and the above is just an example. Details as to why this presentation is correct can be found in the second author's $\mathrm{PhD}$ thesis [26].)

The vertex groups $G_{i}=K / R_{i}$ are generated by any generating set of $K$, in particular by $\{a, b a b\}$. They abide by the relations that generate $R_{i}$ so in the case of $R_{0}$ these are $a^{5}, a b a^{2} b=a(b a b)^{2}$ and $b a^{5} b=(b a b)^{5}$ (when we write them in terms of the generators of $K$ ). So we have

$$
\begin{array}{rlr}
G_{0} & =\left\langle a, b a b \mid a^{5}, a(b a b)^{2},(b a b)^{5}\right\rangle & \\
& =\left\langle b a b \mid(b a b)^{-10},(b a b)^{5}\right\rangle & \text { as } a=(b a b)^{-2} \\
& =\mathbb{Z} / 5 \mathbb{Z}=\langle b a b\rangle &
\end{array}
$$

and similarly

$$
\begin{array}{rlr}
G_{1} & =\left\langle a, b a b \mid(b a b)^{5},(b a b) a^{2}, a^{5}\right\rangle & \\
& =\left\langle a \mid a^{-10}, a^{5}\right\rangle & \text { as }(b a b)=a^{-2} \\
& =\mathbb{Z} / 5 \mathbb{Z}=\langle a\rangle . &
\end{array}
$$

The fact that $G_{0}$ is isomorphic to $G_{1}$ is not a coincidence as we remark at the end of this section. In Fig. 1, the vertices depicted as square correspond to $V_{0}=\tilde{V}_{0} / \sim$, and vertices depicted as circles correspond to $V_{1}=\tilde{V}_{1} / \sim$.

Note that we have made $\mathcal{S}$ a subset of the group $M F_{P}$, and so each $s \in \mathcal{S}$ has an inverse $s^{-1}$ in $M F_{P}$. With these inverses in mind we define $\mathcal{S}^{-1}:=\left\{s^{-1}: s \in \mathcal{S}\right\}$. Note that $s=s^{-1}$ exactly when $s \in \mathcal{I}$. Moreover, as $\mathcal{S}_{1} \subset K$ and $G_{i}=K / R_{i}$, we can think of $\mathcal{S}_{1}$ as a subset of $G_{i}$ in the following proposition:

Proposition 3.4 For every 2-partite presentation $P=\left\langle\mathcal{S}_{1}, \mathcal{U}, \mathcal{I} \mid \mathcal{R}_{0}, \mathcal{R}_{1}\right\rangle$, the subgraph of $\Gamma:=P C a y(P)$ with edges coloured by $\mathcal{S}_{1} \cup \mathcal{S}_{1}^{-1}$ is isomorphic to the disjoint union of $\operatorname{Cay}\left(G_{0}, \mathcal{S}_{1}\right)$ and $\operatorname{Cay}\left(G_{1}, \mathcal{S}_{1}\right)$. 
Proof Let $T_{i}$ be the subgraph of $T_{P}$ induced by the vertices of $\tilde{V}_{i}$, and $\Gamma_{i}$ be the subgraph of $\Gamma$ induced by $V_{i}=\tilde{V}_{i} / \sim$. We will show that $\Gamma_{i}$ is isomorphic to $\operatorname{Cay}\left(G_{i}, \mathcal{S}_{1}\right)$.

To begin with, recall that $\tilde{V}_{0}=K$ and $G_{0}=K / R_{0}$, and so $V_{0}$ is canonically identified with $G_{0}$. Thus to show that $\Gamma_{0}$ is isomorphic to $\operatorname{Cay}\left(G_{0}, \mathcal{S}_{1}\right)$, we need to check that $(v, w)$ is a directed edge of $\Gamma_{0}$ coloured $s$ whenever $w=v s$. The latter holds whenever $v^{\prime} s \in \eta^{-1}(w)$ for every $v^{\prime} \in \eta^{-1}(v)$, which is exactly when $\left(v^{\prime}, v^{\prime} s\right)$ is a directed edge of $T_{P}$ coloured $s$. This in turn is equivalent to $(v, w)$ being a directed edge of $\Gamma_{0}$ coloured $s$ because $c=c^{\prime} \circ \eta$.

This proves that $\Gamma_{0}$ is isomorphic to Cay $\left(G_{0}, \mathcal{S}_{1}\right)$. To prove that $\Gamma_{1}$ is isomorphic to $\operatorname{Cay}\left(G_{1}, \mathcal{S}_{1}\right)$ we repeat the same argument multiplying on the left with a fixed element of $\mathcal{S}_{2}$ throughout. Since $V(\Gamma)$ is the disjoint union of $V_{0}$ and $V_{1}$, our statement follows.

Proposition 3.5 For every 2-partite presentation $P=\left\langle\mathcal{S}_{1}, \mathcal{U}, \mathcal{I} \mid \mathcal{R}_{0}, \mathcal{R}_{1}\right\rangle$, the graph $\Gamma:=P \operatorname{Cay}(P)$ is regular, with vertex degree $\left|\mathcal{S} \cup \mathcal{S}^{-1}\right|=2\left|\mathcal{S}_{1}\right|+2|\mathcal{U}|+|\mathcal{I}|$.

Proof By Proposition 3.4, the subgraph with edges coloured by $\mathcal{S}_{1} \cup \mathcal{S}_{1}^{-1}$ is $2\left|\mathcal{S}_{1}\right|$ regular. It therefore suffices to prove that every vertex in $\Gamma$ has a unique outgoing edge coloured $s$ for every $s \in \mathcal{S}_{2} \cup \mathcal{S}_{2}^{-1}$. Existence is easy by the definition of $T_{P}$. To prove uniqueness, suppose in $T_{P}$ we have two edges $\left(v_{0}, u_{0}\right),\left(v_{1}, u_{1}\right) \in \vec{E}\left(T_{P}\right)$ where $c\left(v_{0}, u_{0}\right)=s=c\left(v_{1}, u_{1}\right)$ and $v_{0} \sim v_{1}$. So by definition $u_{i}=v_{i} s$ and $v_{0}^{-1} v_{1} \in R_{i}$ for $i \in \mathbb{Z} / 2 \mathbb{Z}$. Note that

$$
u_{0}^{-1} u_{1}=s^{-1} v_{0}^{-1} v_{1} s=s^{-1}\left(v_{0}^{-1} v_{1}\right) s \in s^{-1} R_{i} s \subset R_{i+1},
$$

which means that $u_{0} \sim u_{1}$ and hence $\left(v_{0}, u_{0}\right) \sim\left(v_{1}, u_{1}\right)$ proving our uniqueness statement.

Corollary 3.6 For a 2-partite presentation $P=P \operatorname{Cay}\left\langle\mathcal{S}_{1}, \mathcal{U}, \mathcal{I} \mid \mathcal{R}_{0}, \mathcal{R}_{1}\right\rangle$ the universal cover of $\Gamma:=P C a y(P)$ is $T_{P}$. Moreover, every edge with a colour in $\mathcal{S}_{1}$ connects two vertices in $V_{i}$ for some $i \in \mathbb{Z} / 2 \mathbb{Z}$, and every edge with a colour in $\mathcal{S}_{2}$ connects a vertex in $V_{i}$ to a vertex in $V_{i+1}$.

Proof Recall that $\sim$ defines a map of graphs $\eta: T_{P} \rightarrow \operatorname{PCay}(P)$, by $\eta(x)=[x]$. As both $T_{P}$ and $T_{P} / \sim$ are $\left|\mathcal{S} \cup \mathcal{S}^{-1}\right|$-regular by Proposition 3.5, and $\eta$ is locally injective, $\eta$ is a cover. As the fundamental group of a tree is trivial we deduce that $\eta$ is in fact the universal cover.

By Proposition 3.4, edges labelled $\mathcal{S}_{1}$ connect vertices in $G_{i}$ to vertices in $G_{i}$, which are exactly the vertices in $V_{i}$. Moreover, in $T_{P}$ edges labelled $\mathcal{S}_{2}$ connect vertices in $\tilde{V}_{i}$ to $\tilde{V}_{i+1}$. Therefore, edges labelled $\mathcal{S}_{2}$ in $\Gamma$ connect vertices in $\tilde{V}_{i} / \sim=V_{i}$ to vertices in $\tilde{V}_{i+1} / \sim=V_{i+1}$.

\subsection{Topological definition}

We now give an alternative definition of $\Gamma=\operatorname{PCay}(P)$ following the standard topological approach of defining a Cayley graph. 
Let $X$ be a set. Define the rose $\operatorname{Ro}_{X}$ to be a graph with a single vertex $v$ and edge set $E\left(\operatorname{Ro}_{X}\right)=X$, where each $x \in X=E\left(\operatorname{Ro}_{X}\right)$ signifies a loop at $v$. To be more precise, we let $X^{-1}$ denote an abstract set disjoint from $X$ and in bijection (denoted ${ }^{-1}$ ) with $X$, and let $X \cup X^{-1}$ be the set of directed edges of $\operatorname{Ro}_{X}$. The terminus map $\tau$ of $\operatorname{Ro}_{X}$ maps all edges to $v$. We colour this rose by $c: \vec{E}\left(\operatorname{Ro}_{X}\right) \rightarrow X \cup X^{-1}$ by an arbitrary choice of orientation; in other words, $c$ is a bijection from $\vec{E}$ (Rox) to $X \cup X^{-1}$ satisfying $c\left(e^{-1}\right)=c(e)^{-1}$ for every $e \in X$.

For a presentation $P=\langle\mathcal{S} \mid \mathcal{R}\rangle$ of a group one often alternatively defines the Cayley graph in the following more topological way. We start by constructing the presentation complex $\mathcal{C}(P)$ as follows. The 1 -skeleton of $\mathcal{C}(P)$ is Ro $\mathcal{S}$ with vertex $v$. For each relator $r \in \mathcal{R}$, we introduce a 2-cell $D_{r}$ and identify its boundary with the closed walk of RoS dictated by $r$ (see Definition 3.9). This completes the definition of $\mathcal{C}(P)$. The Cayley graph $\operatorname{Cay}\langle\mathcal{S} \mid \mathcal{R}\rangle$ is the 1 -skeleton of the universal cover of $\mathcal{C}(P)$.

We now generalise this construction to the context of our 2-partite presentations. We remark that it is not so easy to obtain the modified Cayley graphs using this construction because $\operatorname{Ro}_{\mathcal{S}}$ has even degree, so any cover will also have even degree. But treating $\mathcal{I}$ appropriately we will in fact be able to obtain graphs of odd degree.

Definition 3.7 Let $P=\left\langle\mathcal{S}_{1}, \mathcal{U}, \mathcal{I} \mid \mathcal{R}_{0}, \mathcal{R}_{1}\right\rangle$ be a 2-partite presentation. We construct the presentation complex $\mathcal{C}(P)$ of $P$ as follows. Start with two copies of Ro $\mathcal{S}_{1}$, with vertices $v_{0}$ and $v_{1}$, respectively, and connect $v_{0}$ and $v_{1}$ with an edge for each element of $\mathcal{S}_{2} \cup \mathcal{S}_{2}^{-1} \subset M F_{P}$. We will refer to this 1-complex $C(P)$ as the presentation graph of $P$. We can extend the colouring of the two copies of Ro $\mathcal{S}_{1}$ to a colouring $c: \vec{E}(C(P)) \rightarrow \mathcal{S} \cup \mathcal{S}^{-1}$ where $c(e)^{-1}=c\left(e^{-1}\right)$.

To define the 2-cells of $\mathcal{C}(P)$, for each relator $r=s_{1} s_{2} \ldots s_{n} \in \mathcal{R}_{i}$, we start a walk $p_{r}$ at $v_{i}$ and extend this walk inductively with the edge labelled $s_{i}, i=1, \ldots, n$. The path $p_{r}$ starts and ends at $v_{i}$ as $\mathcal{R}_{i} \subset K$. Attach a 2-cell along each such closed walk $p_{r}$ to obtain the presentation complex $\mathcal{C}(P)$ from $C(P)$. Finally, we define the (topological) 2-partite Cayley graph $\mathrm{PCay}_{T}\left\langle\mathcal{S}_{1}, \mathcal{U}, \mathcal{I} \mid \mathcal{R}_{0}, \mathcal{R}_{1}\right\rangle$ to be the 1-skeleton of the universal cover of $\mathcal{C}(P)$.

Our next result, Theorem 3.11, says that this gives rise to the same graph as in Definition 3.2. To prove it, we will use the theory of covering spaces (Sect. 2.4). For this we need to turn our graphs into topological spaces, and we now recall the standard way to do so.

Given a graph $\Gamma$ with vertex set $V$, and any orientation on its edges $O \subset \vec{E}(\Gamma)$, we define a topological space as follows. Associate a point to each vertex, and a closed interval $I_{e}=[0,1]$ to each edge $e \in O$. Then define the quotient $I_{e}(0) \sim \tau\left(e^{-1}\right)$ and $T_{e}(1) \sim \tau(e)$ to obtain the topological space

$$
\Gamma=\left(V \cup \bigcup_{e \in O} I_{e}\right) / \sim .
$$

It is not hard to see that when $\Gamma$ is connected this topological space is path-connected, locally path-connected and semilocally simply-connected. Moreover, different choices of $O$ define homeomorphic topological spaces. 
Next, we introduce a notion of edge-colouring that will be useful to establish that certain maps of graphs are covers. Recall that a Cayley graph can be naturally edgecoloured using the set of generators as colours. The $C$ ayley-like colourings we now define imitate, and extend, this colouring.

Definition 3.8 Let $\Gamma$ be a graph with a colouring $c: \vec{E}(\Gamma) \rightarrow X$. We say that $c$ is Cayley-like, if

1. $\Gamma$ is $|X|$-regular,

2. for all $e, e^{\prime} \in \vec{E}(\Gamma)$, if $c(e)=c\left(e^{\prime}\right)$ and $\tau(e)=\tau\left(e^{\prime}\right)$ then $e=e^{\prime}$, and

3. there is an involution ${ }^{-1}: X \rightarrow X$ such that $c(e)^{-1}=c\left(e^{-1}\right)$.

Suppose we have two graphs $\Gamma$ and $\Delta$ with Cayley-like colourings $c_{\Gamma}: \vec{E}(\Gamma) \rightarrow X$ and $c_{\Delta}: \vec{E}(\Delta) \rightarrow X$. Then any surjective map of graphs $\phi: \Gamma \rightarrow \Delta$ which respects these colourings, that is, satisfies $c_{\Gamma}=c_{\Delta} \circ \phi$, is a covering map of the associated topological spaces. Indeed, $\phi$ can't map any two edges that share an end vertex to the same edge, as this cannot respect the colourings.

Let $\mathcal{P}_{v}(\Gamma)$ be the set of walks in $\Gamma$ starting at a vertex $v$, and define the group $M F_{X}$ by the presentation $\left\langle X \mid\left\{x x^{-1}: x \in X\right\}\right\rangle$. Then any Cayley-like colouring $c: \vec{E}(\Gamma) \rightarrow X$ defines a map $\mathcal{W}_{v}: \mathcal{P}_{v}(\Gamma) \rightarrow M F_{X}$ by $p=v e_{1} v_{1} \ldots e_{n} v_{n} \mapsto c\left(e_{1}\right) c\left(e_{2}\right) \ldots c\left(e_{n}\right)$. Note that there is a well-defined inverse $\mathcal{W}_{v}^{-1}: M F_{X} \rightarrow \mathcal{P}_{v}(\Gamma)$ as at every vertex $v^{\prime} \in V(\Gamma)$ there is a unique edge $e \in \vec{E}(\Gamma)$ with colour $c(e)$ and $\tau\left(e^{-1}\right)=v^{\prime}$. Moreover, $\mathcal{W}_{v}^{-1}$ is a double-sided inverse to $\mathcal{W}_{v}$, so both these maps are bijections.

Definition 3.9 For any $g \in M F_{X}$, we say that $\mathcal{W}_{v}^{-1}(g)$ is the walk (in $\Gamma$ ) dictated by the word $g$ starting at $v$.

This is a natural definition since we can express $g$ as a word $s_{1} \ldots s_{n}$ with $s_{i} \in X \cup X^{-1}$, and obtain $\mathcal{W}_{v}^{-1}(g)$ by starting at $v$ and following the directed edges with colours $c\left(s_{1}\right) \ldots c\left(s_{n}\right)$; this is well-defined when $c$ is Cayley-like.

It is straightforward to check that if $p$ is homotopic to $p^{\prime}$, then $\mathcal{W}_{v}(p)=\mathcal{W}_{v}\left(p^{\prime}\right)$. Thus by restricting to the closed walks we can think of $\mathcal{W}_{v}$ as a map from $\pi_{1}(\Gamma, v)$ to $M F_{X}$, and so the above remarks imply that

Proposition $3.10 \mathcal{W}_{v}$ is a group isomorphism from $\pi_{1}(\Gamma, v)$ to a subgroup of $M F_{X}$.

Suppose we have a covering map of graphs $\psi: \Delta \rightarrow \Gamma$ both of which have Cayleylike colourings $c_{\Delta}: \vec{E}(\Delta) \rightarrow X$ and $c_{\Gamma}: \vec{E}(\Gamma) \rightarrow X$ such that $c_{\Delta}=c_{\Gamma} \circ \psi$. For a path $p:[0,1] \rightarrow \Gamma$ with $p(0), p(1) \in V(\Gamma)$ and a lift $\tilde{p}:[0,1] \rightarrow \Delta$ of $p$ by $\psi$, it is straightforward to check that

$$
\mathcal{W}_{p(0)}(p)=\mathcal{W}_{\tilde{p}(0)}(\tilde{p})
$$

where with a slight abuse, we interpreted $p$ as a walk in $\Gamma$ in the obvious way. We are now ready to prove that our two definitions of $\operatorname{PCay}(P)$ coincide:

Theorem 3.11 For every 2-partite presentation $P=\left\langle\mathcal{S}_{1}, \mathcal{U}, \mathcal{I} \mid \mathcal{R}_{0}, \mathcal{R}_{1}\right\rangle$, the 2-partite Cayley graphs $\Gamma=P C a y(P)$ and $\Delta=\operatorname{PCay}_{T}(P)$ are isomorphic. 

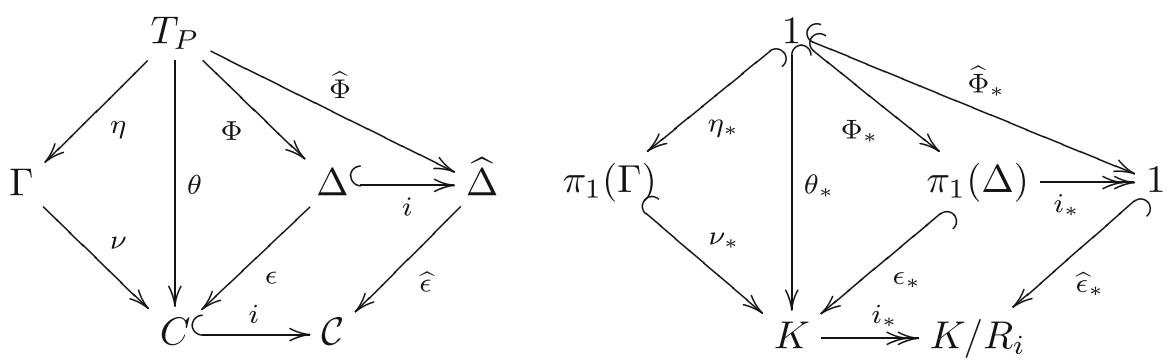

Fig. 2 Maps used in Proposition 3.11

Proof Our presentation graph $C=C(P)$ is $\left|\mathcal{S} \cup \mathcal{S}^{-1}\right|$-regular by definition. Therefore, the universal cover of $C$ is the $\left|\mathcal{S} \cup \mathcal{S}^{-1}\right|$-regular tree $T$, and we can let $\theta: T \rightarrow C$ be the corresponding covering map. Let $c_{C}: \vec{E}(C) \rightarrow \mathcal{S} \cup \mathcal{S}^{-1}$ be the colouring of $C$ as above. This lifts to a colouring $c_{T}: \vec{E}(T) \rightarrow \mathcal{S} \cup \mathcal{S}^{-1}$ of $T$, by letting $c_{T}(e):=c_{C}(\theta(e))$. This colouring allows us to identify $T$ with $T_{P}$.

Let $p \in \pi_{1}\left(C, v_{i}\right)$. As $c_{C}$ is a Cayley-like colouring of $C$, we can consider $\mathcal{W}_{v_{i}}(p) \in$ $M F_{P}$ by Definition 3.8 and the discussion thereafter. Any closed walk representing $p$ must use an even number of edges coloured $\mathcal{S}_{2} \cup \mathcal{S}_{2}^{-1}$ by the definition of $C$, so $\mathcal{W}_{v_{i}}(p) \in K \subset M F_{P}$. Moreover, each $k \in K$ gives rise to a closed walk $\mathcal{W}_{v_{i}}^{-1}(k)$ representing some element of $\pi_{1}\left(C, v_{i}\right)$. Thus by Proposition 3.10,

$$
\mathcal{W}_{v_{i}} \text { is an isomorphism from } \pi_{1}\left(C, v_{i}\right) \text { onto } K \text {. }
$$

Recall that we can identify $T$ with $T_{P}$. If in doing so we identify the identity $1_{M F_{P}} \in$ $V\left(T_{P}\right)$ of $M F_{P}$ with some vertex in $\theta^{-1}\left(v_{0}\right)$ (which we easily can) then (2) implies

$$
\theta\left(\tilde{V}_{i}\right)=v_{i}
$$

because $\tilde{V}_{0}=K$ and $\tilde{V}_{1}=\mathcal{S}_{2} K$.

Let $\eta: T_{P} \rightarrow \Gamma$ be the covering map found in Corollary 3.6. Let $c_{\Gamma}: \vec{E}(\Gamma) \rightarrow$ $\mathcal{S} \cup \mathcal{S}^{-1}$ be the colouring of $\Gamma$ as in its definition. Now define a map $v: \Gamma \rightarrow C$ by letting $v(v)=v_{i}$ whenever $v \in V_{i}=\eta\left(\tilde{V}_{i}\right)$. If $c_{\Gamma}(e)=s$ for some $e \in \vec{E}(\Gamma)$ then $v$ maps $e$ to the unique edge $e^{\prime} \in \vec{E}(C)$ with $c_{C}\left(e^{\prime}\right)=s$ and $\tau\left(e^{\prime}\right)=v(\tau(e))$. Since for every $v \in \tilde{V}_{i}$ we have $\eta(v) \in V_{i}$, we have $v(\eta(v))=v_{i}$ and hence $\theta=v \circ \eta$ by (3).

Let $\widehat{\epsilon}: \widehat{\Delta} \rightarrow \mathcal{C}$ be the universal cover of $\mathcal{C}:=\mathcal{C}(P)$. We know that $\Delta$ and $C$ are the 1 -skeletons of $\widehat{\Delta}$ and $\mathcal{C}$, respectively, so we obtain the inclusion maps $i: \Delta \rightarrow \widehat{\Delta}$ and $i: C \rightarrow \mathcal{C}$. Furthermore, by restricting $\widehat{\epsilon}$ to the 1 -skeleton we obtain a covering map $\epsilon: \Delta \rightarrow C$. As $\theta: T_{P} \rightarrow C$ is the universal cover of $C$, it can be lifted through $\epsilon: \Delta \rightarrow C$ to a map $\Phi: T_{P} \rightarrow \Delta$ so that $\epsilon \circ \Phi=\theta$ by the definition of a universal cover. This gives us a map $\widehat{\Phi}: T_{P} \rightarrow \widehat{\Delta}$ defined by $\widehat{\Phi}:=i \circ \Phi$. Note that all these maps respect the colourings of the edges as $\theta$ and $\widehat{\epsilon}$ do.

By Theorem 2.1, to show $\Gamma \cong \Delta$ it suffices to show that $\nu_{*}\left(\pi_{1}(\Gamma)\right)=\epsilon_{*}\left(\pi_{1}(\Delta)\right)$, or equivalently $\mathcal{W}_{v_{i}}\left(v_{*}\left(\pi_{1}(\Gamma)\right)\right)=\mathcal{W}_{v_{i}}\left(\epsilon_{*}\left(\pi_{1}(\Delta)\right)\right)$ as $\mathcal{W}_{v_{i}}$ is a bijection. To do so, 
we will prove that the latter groups are both equal to $R_{i}$, where $R_{i}$ is as defined after Definition 3.1 .

To show that $\mathcal{W}_{v_{i}}\left(v_{*}\left(\pi_{1}(\Gamma)\right)\right)=R_{i}$, let $p$ be a closed walk representing some element of $\pi_{1}(\Gamma, v)$ with $v \in V_{i}$. Choose a lift of $p$ to a walk $\tilde{p}:[0,1] \rightarrow T_{P}$ (so $\eta \circ \tilde{p}=p$ ). We know that $\eta(\tilde{p}(0))=\eta(\tilde{p}(1))=v$, so $\tilde{p}(0), \tilde{p}(1) \in \eta^{-1}(v)$ implying $\tilde{p}(0)^{-1} \tilde{p}(1) \in R_{i}$. So $\mathcal{W}_{v_{i}}\left(v_{*}(p)\right)=\mathcal{W}_{v_{i}}(\theta(\tilde{p})) \in R_{i}$, which proves that $\mathcal{W}_{v_{i}}\left(v_{*}\left(\pi_{1}(\Gamma)\right)\right) \subseteq R_{i}$

We would like to use Proposition 3.10 to deduce $\mathcal{W}_{v_{i}}\left(\nu_{*}\left(\pi_{1}(\Gamma)\right)\right)=R_{i}$, and for this it now only remains to prove that the former is surjective onto $R_{i}$. To show this, pick any $r \in R_{i}$. As $R_{i} \subset K \cong \mathcal{W}_{v_{i}}\left(\pi_{1}\left(C, v_{i}\right)\right)$ by (2), there is a representative $q$ of an element of $\pi_{1}\left(C, v_{i}\right)$ such that $\mathcal{W}_{v_{i}}(q)=r$. Choose a lift $\tilde{q}:[0,1] \rightarrow T_{P}$ of $q$ through $v \circ \eta=\theta$, such that $\eta(\tilde{q}(0))=v$ (and so $v \circ \eta \circ \tilde{q}=\theta \circ \tilde{q}=q$ ). Then as $\mathcal{W}_{v}(\tilde{q})=\mathcal{W}_{v_{0}}(q)=r \in R_{i}$ we have $\tilde{q}(0)^{-1} \tilde{q}(1) \in R_{i}$, and so $\tilde{q}(0) \sim \tilde{q}(1)$, with $\sim$ as in the definition of $\Gamma$ as a quotient of $T_{P}$. This means that $\eta(\tilde{q}(1))=\eta(\tilde{q}(0))=v$, and so $\eta \circ \tilde{q}$ is a loop representing an element of $\pi_{1}(\Gamma, v)$. Since $v_{*}(\eta \circ \tilde{q})=\theta \circ \tilde{q}=q$ represents an element of $\nu_{*}\left(\pi_{1}(\Gamma)\right)$ we deduce that $r=\mathcal{W}_{v_{i}}(q) \in \mathcal{W}_{v_{i}}\left(\nu_{*}\left(\pi_{1}(\Gamma, v)\right)\right)$, proving that $\mathcal{W}_{v_{i}}\left(v_{*}\left(\pi_{1}(\Gamma, v)\right)\right)$ surjects onto $R_{i}$ as desired.

Next, we prove $\mathcal{W}_{v}\left(\epsilon_{*}\left(\pi_{1}(\Delta, v)\right)\right) \subseteq R_{i}$ for every $v \in V(\Delta)$ with $\epsilon(v)=v_{i}$. It is well-known [13, Proposition 1.26] that the inclusion of the one skeleton into a 2-simplex induces a surjection on the level of fundamental groups, and the kernel is exactly the normal closure of the words bounding the 2-cells. Thus $i_{*}: \pi_{1}\left(C, v_{i}\right) \rightarrow$ $\pi_{1}\left(\mathcal{C}, v_{i}\right)$ is a surjection. Combining these remarks with (2), it follows that $i_{*} \circ \mathcal{W}_{v_{i}}^{-1}$ : $K \rightarrow \pi_{1}\left(\mathcal{C}, v_{i}\right)$ is a surjection, with kernel $R_{i}$, since $R_{i}$ is the normal closure in $K$ of the words onto which $\mathcal{W}_{v_{i}}^{-1}$ maps the closed walks bounding 2-cells of $\mathcal{C}$ by the definition of $\mathcal{C}$. Thus $\pi_{1}\left(\mathcal{C}, v_{i}\right)=K / R_{i}=G_{i}$. Now pick $v \in V(\Delta)$ with $\epsilon(v)=v_{i}$. As $i \circ \epsilon=\widehat{\epsilon} \circ i$ and $\pi_{1}(\widehat{\Delta})=1$, we have $\left(i_{*} \circ \epsilon_{*}\right)\left(\pi_{1}(\Delta, v)\right)=\left(\widehat{\epsilon}_{*} \circ i_{*}\right)\left(\pi_{1}(\Delta, v)\right)=1$, and so $\mathcal{W}_{v}\left(\epsilon_{*}\left(\pi_{1}(\Delta, v)\right)\right) \leq \operatorname{ker}\left(i_{*}\right)=R_{i}$ as desired.

Finally, we claim that $R_{i} \subset \mathcal{W}_{v}\left(\epsilon_{*}\left(\pi_{1}(\Delta, v)\right)\right)$ for every $v \in V(\Delta)$ with $\epsilon(v)=v_{i}$. For this, pick $r \in R_{i}$, and note that as $R_{i} \subset K$ and $K \cong \mathcal{W}_{v_{i}}\left(\pi_{1}\left(C, v_{i}\right)\right)$ by (2), there is a representative $t$ of an element of $\pi_{1}\left(C, v_{i}\right)$ such that $\mathcal{W}_{v_{i}}(t)=r$. We can write $\mathcal{W}_{v_{i}}(t)=r=\prod_{j=1}^{n} w_{j} r_{j} w_{j}^{-1} \in M F_{P}$ for $w_{j} \in K$ and $r_{j} \in \mathcal{R}_{i} \cup s \mathcal{R}_{i+1} s^{-1}$ with $s \in \mathcal{S}_{2} \cup \mathcal{S}_{2}^{-1}$ by the definition of $R_{i}$. Choose a lift $t^{\prime}:[0,1] \rightarrow \Delta$ of $t$ through $\epsilon$ so that $t^{\prime}(0)=v$. By (1) we have $\mathcal{W}_{v_{i}}(t)=\mathcal{W}_{v}\left(t^{\prime}\right)$. Note that $\mathcal{W}_{v}^{-1}\left(w_{j} r_{j} w_{j}^{-1}\right)$ is a loop of $\Delta$ as $\mathcal{W}^{-1}\left(r_{j}\right)$ is contractable in $\widehat{\Delta}$, and so it represents some element of $\pi_{1}(\Delta, v)$. Applying this to each factor of our above expression $r=\prod_{j=1}^{n} w_{j} r_{j} w_{j}^{-1}$ implies that $t^{\prime}$ represents some element of $\pi_{1}(\Delta, v)$. Thus $\mathcal{W}_{v_{i}}\left(\epsilon_{*}\left(t^{\prime}\right)\right)=\mathcal{W}_{v_{i}}(t)=r$, which means that $R_{i} \subset \mathcal{W}_{v}\left(\epsilon_{*}\left(\pi_{1}(\Delta, v)\right)\right)$ as claimed.

To summarise, we have proved that $\mathcal{W}_{v_{i}}\left(\nu_{*}\left(\pi_{1}(\Gamma)\right)\right)=R_{i}=\mathcal{W}_{v_{i}}\left(\epsilon_{*}\left(\pi_{1}(\Delta)\right)\right)$, implying that $\Gamma \cong \Delta$. Moreover, it is straightforward to check that as all the maps above respect the edge colourings, so does this isomorphisms of graphs.

From now on we just use the notation $\operatorname{PCay}(P)$ for the 2-partite Cayley graph obtained in either Definition 3.2 or 3.7. 
As a corollary of the above proof, we deduce that the covers $v, \epsilon$ are equal, and so

$$
V_{i}=v^{-1}\left(v_{i}\right)=\epsilon^{-1}\left(v_{i}\right)
$$

and similarly $V_{i}=\eta\left(\tilde{V}_{i}\right)=\Phi\left(\tilde{V}_{i}\right)$, so $V_{i}$ is well defined for either the topological or graph definition, as in the notation of Fig. 2. From now on we will only use $\epsilon$ to denote this covering map.

The following corollary gathers some further facts that we obtained in the proof of Theorem 3.11 for future reference.

Corollary 3.12 Let $P=\left\langle\mathcal{S}_{1}, \mathcal{U}, \mathcal{I} \mid \mathcal{R}_{0}, \mathcal{R}_{1}\right\rangle$ be a 2-partite presentation with partite Cayley graph $\Gamma:=P C a y(P)$. We have

1. $\pi_{1}\left(\mathcal{C}(P), v_{i}\right)$ is isomorphic to $G_{i}$;

2. $\mathcal{W}_{v_{i}}$ is an isomorphism from $\pi_{1}\left(C(P), v_{i}\right)$ onto $K$;

3. $\mathcal{W}_{v_{i}}$ is an isomorphism from $\pi_{1}(\Gamma, v)$ onto $R_{i}$ for every $v \in V_{i}$; and

4. the sequence $0 \rightarrow \pi_{1}(\Gamma, v) \stackrel{\epsilon_{*}}{\rightarrow} \pi_{1}\left(C(P), v_{i}\right) \stackrel{i_{*}}{\rightarrow} \pi_{1}\left(\mathcal{C}(P), v_{i}\right) \rightarrow 0$ is exact, where $\epsilon: \Gamma \rightarrow C(P)$ is the cover in Definition 3.7, and $i: C(P) \rightarrow \mathcal{C}(P)$ the inclusion.

The generalised Petersen graph is denoted by $P(n, k)$ and defined as follows. Let

$$
\begin{aligned}
& V(P(n, k)):=\left\{x_{i}, y_{i} \mid i \in \mathbb{Z} / n \mathbb{Z}\right\}, \text { and } \\
& E(P(n, k)):=\left\{\left(x_{i}, x_{i+1}\right),\left(x_{i}, y_{i}\right),\left(y_{i}, y_{i+k}\right) \mid i \in \mathbb{Z} / n \mathbb{Z}\right\} .
\end{aligned}
$$

The classical example is the Petersen graph, $P(5,2)$, the smallest non-Cayley vertextransitive graph. The following statement, proved in the second author's PhD thesis [26], says that we can obtain every $P(n, k)$ as a 2-partite Cayley graph.

Theorem 3.13 The generalised Petersen graph $P(n, k)$ is isomorphic to $P C a y\left\langle\{a\}, \varnothing,\{b\} \mid\left\{a^{n}, a b a^{k} b\right\},\left\{a^{n}\right\}\right\rangle$.

Note that from the definition of $R_{i}$ we have $R_{0}=s R_{1} s^{-1}$ for any $s \in \mathcal{S}_{2}$. Therefore, we deduce that $G_{i}:=R_{i} \backslash K \cong R_{i+1} \backslash K$, where an isomorphism $\phi_{s, i}: G_{i} \rightarrow G_{i+1}$ is given by conjugation by any $s \in \mathcal{S}_{2}$. This property isn't enough to guarantee vertex transitivity of $\Gamma$, with a counter example given by $P(4,2)$. This invites the following rather vague question.

Question 3.14 For which 2-partite presentations $P$ is $P C a y(P)$ vertex-transitive?

We know that PCay $(P)$ is not always vertex-transitive, see e.g. Fig. 6.

\section{Relationships to Bi-Cayley and Haar graphs}

We recall that an action on a graph $\Gamma$ is semi-regular (or free) if $g \cdot x=h \cdot x$ implies $g=h$ for every $g, h \in G$ and $x \in V(\Gamma)$. A vertex-transitive graph $\Gamma$ is said to be $n$ Cayley over $G$ if $\mathrm{G}$ is a semi-regular subgroup of $\operatorname{Aut}(\Gamma)$ with $n$ orbits of vertices. If $n=2$ we say that $\Gamma$ is bi-Cayley. 
Fig. 3 The labelling of the Petersen graph used in Example 4.1

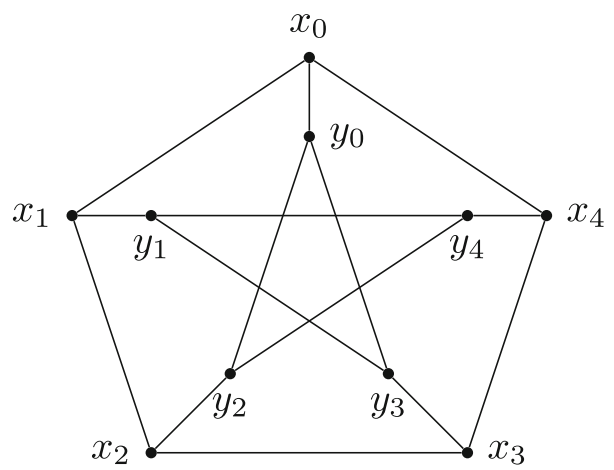

Suppose $\Gamma$ is bi-Cayley over G. Pick two vertices $e_{0}, e_{1} \in V(\Gamma)$ from different orbits of $G$. As $G$ has exactly two orbits in $V(\Gamma)$, and it acts regularly on each of them, for any $x \in V(\Gamma)$ there exists a unique $i \in \mathbb{Z} / 2 \mathbb{Z}$ and $g \in G$ such that $g \cdot e_{i}=x$, so we define $x=:(g)_{i}$. Each of the two orbits $O_{i}:=\left\{(g)_{i}: g \in G\right\}$ forms a (possibly disconnected) Cayley graph of $G$ with respect to the generating sets $R=R^{-1}=$ $\left\{g \in G \mid e_{0}(g)_{0} \in E(\Gamma)\right\}$ and $L=L^{-1}=\left\{g \in G \mid e_{1}(g)_{1} \in E(\Gamma)\right\}$, respectively. Here, by $v w \in E(\Gamma)$ we mean that either $(v, w) \in \vec{E}(\Gamma)$ or $(w, v) \in \vec{E}(\Gamma)$. To capture the set $E_{01}$ of edges of the form $(g)_{0}(h)_{1} \in E(\Gamma)$, we introduce the set $S=\left\{g \in G \mid\left(e_{0},(g)_{1}\right) \in \vec{E}(\Gamma)\right\}$ and note that $S$ uniquely determines $E_{01}$ as any $e \in E_{01}$ coincides with $h \cdot e_{0}(g)_{1}$ for some $g \in S$ and $h \in G$.

To summarise, we can represent any bi-Cayley graph $\Gamma$ over $\operatorname{Gas} \operatorname{BiCay}(G, R, L, S)$ where $R, L, S \subset G$ with $R=R^{-1}$ and $L=L^{-1}$. Then the set of directed edges of $\Gamma=: \operatorname{BiCay}(G, R, L, S)$ is

$$
\begin{aligned}
& \left\{\left((g)_{0},(g r)_{0}\right) \mid g \in G, r \in R\right\} \cup\left\{\left((g)_{1},(g l)_{1}\right) \mid g \in G, l \in L\right\} \\
& \quad \cup\left\{\left((g)_{0},(g s)_{1}\right) \mid g \in G, s \in S\right\} \cup\left\{\left((g)_{1},\left(g s^{-1}\right)_{0}\right) \mid g \in G, s \in S\right\} .
\end{aligned}
$$

This representation isn't unique: if we choose different vertices for $e_{0}, e_{1}$ or a different action of $G$ we potentially obtain different sets $R, S$ and $L$. Note that $\operatorname{BiCay}(G, R, L, S)$ is regular if and only if $|R|=|L|$.

Example 4.1 Consider again the Petersen graph $\Gamma=P(5,2)$ as in Example 3.3 (Fig. 3). This has a natural action of $G:=\mathbb{Z} / 5 \mathbb{Z}=<a>$ where

$$
a^{j}: \begin{gathered}
x_{i} \\
y_{i}
\end{gathered}{\stackrel{x}{x_{i+j}}}_{y_{i+j}}
$$

To represent this as a bi-Cayley graph with above notation, we could choose $\left(a^{0}\right)_{0}:=x_{0}$ and $\left(a^{0}\right)_{1}:=y_{0}$. Then we obtain $R=\left\{a, a^{4}\right\}, L=\left\{a^{2}, a^{3}\right\}$ and $S=\left\{a^{0}\right\}$. If instead we chose $\left(a^{0}\right)_{1}:=y_{1}$ we would obtain $R=\left\{a, a^{4}\right\}, L=\left\{a^{2}, a^{3}\right\}$ and $S=\left\{a^{4}\right\}$.

Recall that we have endowed $\Gamma:=\operatorname{PCay}\left\langle\mathcal{S}_{1}, \mathcal{U}, \mathcal{I} \mid \mathcal{R}_{0}, \mathcal{R}_{1}\right\rangle$ with a colouring $c$ : $\vec{E}(\Gamma) \rightarrow \mathcal{S} \cup \mathcal{S}^{-1}$. We want to talk about automorphisms that preserve this colouring. 
The following definition distinguishes between preserving these colours globally or locally.

Definition 4.2 Let $\Gamma$ be a graph with a colouring $c: \vec{E}(\Gamma) \rightarrow X$. We define the following two subgroups of $\operatorname{Aut}(\Gamma)$ :

$$
\begin{aligned}
\operatorname{Aut}_{c}(\Gamma) & =\{\phi \in \operatorname{Aut}(\Gamma) \mid c(e)=c(\phi(e)) \text { for every } e \in \vec{E}(\Gamma)\}, \text { and } \\
\operatorname{Aut}_{c-l o c}(\Gamma) & =\{\phi \in \operatorname{Aut}(\Gamma) \mid c(x, y)=c(y, z) \Leftrightarrow c(\phi(x, y)) \\
& =c(\phi(y, z)) \text { for all }(x, y),(y, z) \in \vec{E}(\Gamma)\} .
\end{aligned}
$$

We remark that for any 2-partite presentation $P$, there is a subgroup of $\operatorname{Aut}_{c}(\mathrm{PCay}(P))$ witnessing that $\mathrm{PCay}(P)$ is a bi-Cayley graph:

Proposition 4.3 For every 2-partite presentation $P=\left\langle\mathcal{S}_{1}, \mathcal{U}, \mathcal{I} \mid \mathcal{R}_{0}, \mathcal{R}_{1}\right\rangle$ the vertex group $G_{i}$ is a subgroup of $A_{u t}(P C a y(P))$. Moreover, $G_{i}$ acts regularly on $V_{i}$ (and on $\left.V_{i+1}\right)$ for $i \in \mathbb{Z} / 2 \mathbb{Z}$, and so $P C a y(P)$ is bi-Cayley over $G_{0} \cong G_{1}$.

Proof Recall that for a covering map $\eta: X \rightarrow Y$, the group of automorphisms $f: X \rightarrow X$ such that $\eta \circ f=\eta$ is called the deck group of $\eta$ and is denoted by $\operatorname{Aut}(\eta)$. It is known that if $\eta$ is a universal cover $\operatorname{Aut}(\eta)=\pi_{1}(Y)$, and if $X$ is connected and locally path connected then $\operatorname{Aut}(\eta)$ acts freely on $\eta^{-1}(y)$ for any $y \in Y$ [13].

Let $\Gamma:=\operatorname{PCay}(P)$ and let $\widehat{\eta}: \widehat{\Gamma} \rightarrow \mathcal{C}$ be the universal cover of the presentation complex $\mathcal{C}(P)$ of $P$. Thus $\operatorname{Aut}(\widehat{\eta}) \cong \pi_{1}(\mathcal{C}(P)) \cong G_{i}$ by the above remark and Corollary 3.12 1. As $\Gamma$ is the 1-skeleton of $\widehat{\Gamma}$ by Definition 3.7, we can think of $\operatorname{Aut}(\widehat{\eta}) \cong G_{i}$ as a subgroup of $\operatorname{Aut}(\Gamma)$. Moreover, as elements of $\operatorname{Aut}(\widehat{\eta}) \cong G_{i}$ preserve the cover, they preserve the colouring $c: \vec{E}(\Gamma) \rightarrow \mathcal{S} \cup \mathcal{S}^{-1}$ obtained by lifting our colouring of $\mathcal{C}(P)$ via $\widehat{\eta}$, and so we have realised $G_{i}$ as a subgroup of $\operatorname{Aut}_{C}(\Gamma)$. As $\mathcal{C}(P)$ is a connected 2-complex it is locally path connected, therefore $G_{i}$ acts freely on $\widehat{\eta}^{-1}\left(v_{i}\right)=V_{i}$ by the above remarks.

Proposition 4.4 Every regular connected bi-Cayley graph BiCay $(G, R, L, S)$ where $R \cap R^{-1}=L \cap L^{-1}=\emptyset$ and $|R|=|L|$ can be constructed as a 2-partite Cayley graph.

Proof Let $\Gamma:=\operatorname{BiCay}(G, R, L, S)$ be a bi-Cayley graph, and recall our representation of its vertex set as $V(\Gamma)=\left\{(g)_{i} \mid g \in G, i \in \mathbb{Z} / 2 \mathbb{Z}\right\}$. Choose $\mathcal{S}_{1} \subset R$ such that $\mathcal{S}_{1} \cap \mathcal{S}_{1}^{-1}=\varnothing$ and yet $\mathcal{S}_{1} \cup \mathcal{S}_{1}^{-1}=R$. Choose a bijection $f: L \rightarrow R$ such that $f\left(s^{-1}\right)=f(s)^{-1}$. We use $f$ to define the colouring $c: \vec{E}(\Gamma) \rightarrow \mathcal{S}_{1} \cup \mathcal{S}_{1}^{-1} \cup S$ as follows:

$$
\begin{array}{cccc}
\left((g)_{0},(r g)_{0}\right) & r & r & \in R \\
c: \quad\left((g)_{1},(l g)_{1}\right) & \mapsto f(l) & \text { for } & l \in L \\
\left((g)_{0},(s g)_{1}\right)^{ \pm 1} & s & & s \in S .
\end{array}
$$

Note that this colouring is Cayley-like, as there is a unique edge of each colour incident with each vertex. Let $\mathcal{I}:=S$, and set $\mathcal{R}_{0}:=\mathcal{W}_{\left(1_{G}\right)_{0}}\left(\pi_{1}\left(\Gamma,\left(1_{G}\right)_{0}\right)\right)$. We have thus constructed a 2-partite presentation $P:=\left\langle\mathcal{S}_{1}, \emptyset, \mathcal{I} \mid \mathcal{R}_{0}, \emptyset\right\rangle$. We claim that $\Gamma \cong \operatorname{PCay}(P)$. 
To see this, let as usual $\mathcal{C}(P)=: \mathcal{C}$ be the presentation complex and $C(P)=: C$ the presentation graph with vertices $v_{i}, i \in \mathbb{Z} / 2 \mathbb{Z}$ and edges $\vec{E}(C)=\left\{r\left(v_{i}, v_{i}\right), s\left(v_{i}, v_{i+1}\right) \mid r \in\right.$ $\left.\mathcal{S}_{1} \cup \mathcal{S}_{1}^{-1}, s \in S=\mathcal{I}\right\}$ where $c_{C}\left(x\left(v_{i}, v_{j}\right)\right)=x$. We will prove $\Gamma \cong \operatorname{PCay}(P)$ by applying Theorem 2.1 to a cover $\eta: \Gamma \rightarrow C$ defined by $\eta:(g)_{i} \mapsto v_{i}$, and

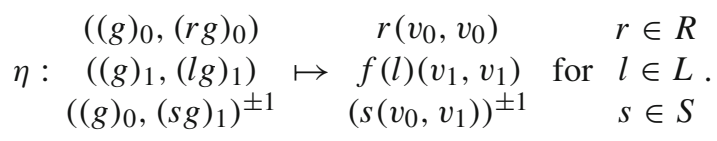

As $\eta$ is a map of graphs with Cayley-like colourings, and $\eta$ respects these colourings by definition, it is indeed a cover. We have $\mathcal{W}_{v_{0}}\left(\eta_{*}\left(\pi_{1}\left(\Gamma,\left(1_{G}\right)_{0}\right)\right)\right)=\mathcal{R}_{0}$ by the choice of $\mathcal{R}_{0}$. Let $\epsilon: \operatorname{PCay}(P) \rightarrow C$ represent the cover given in Definition 3.7 of $\operatorname{PCay}(P)$ (as in Fig. 2). By Corollary $3.12(3)$ we have that $\mathcal{W}_{v_{0}}\left(\epsilon_{*}\left(\pi_{1}(\operatorname{PCay}(P)), v\right)\right)=$ $R_{0}:=\left\langle\left\langle\mathcal{R}_{0}\right\rangle\right\rangle_{K}$ for some $v \in V(\operatorname{PCay}(P))$ such that $\epsilon(v)=v_{0}$. Note that for any $k \in K$ the path $\mathcal{W}_{\left(1_{G}\right)_{0}}^{-1}(k)$ connects $\left(1_{G}\right)_{0}$ to $(g)_{0}$ for some $g \in G$ because it uses an even number of edges $e$ with $c(e) \in S$. This implies

$$
\mathcal{W}_{\left(1_{G}\right)_{0}}^{-1}(k) \pi_{1}\left(\Gamma,(g)_{0}\right) \mathcal{W}_{\left(1_{G}\right)_{0}}^{-1}(k)^{-1}=\pi_{1}\left(\Gamma,\left(1_{G}\right)_{0}\right)
$$

As there exists a colour preserving automorphism of $\Gamma$ mapping $\left(1_{G}\right)_{0}$ to $(g)_{0}$, namely $g$, we moreover have

$$
\mathcal{W}_{(g)_{0}}\left(\pi_{1}\left(\Gamma,(g)_{0}\right)\right)=\mathcal{W}_{\left(1_{G}\right)_{0}}\left(\pi_{1}\left(\Gamma,\left(1_{G}\right)_{0}\right)\right)
$$

Therefore, $\left\langle\left\langle\mathcal{R}_{0}\right\rangle\right\rangle_{K}=\mathcal{R}_{0}$ by (5), (6) and the definition of $\mathcal{R}_{0}$. Using this we have $\mathcal{W}_{v_{0}}\left(\epsilon_{*}\left(\pi_{1}(\operatorname{PCay}(P), v)\right)\right)=\mathcal{R}_{0}$. Moreover, we have $\mathcal{W}_{v_{0}}\left(\eta_{*}\left(\pi_{1}\left(\Gamma,\left(1_{G}\right)_{0}\right)\right)\right)=$ $\mathcal{R}_{0}$ by the definition of $\mathcal{R}_{0}$ and (1). Therefore, $\mathcal{W}_{v_{0}}\left(\epsilon_{*}\left(\pi_{1}(\operatorname{PCay}(P), v)\right)\right)=$ $\mathcal{W}_{v_{0}}\left(\eta_{*}\left(\pi_{1}\left(\Gamma,\left(1_{G}\right)_{0}\right)\right)\right)$, and so by Theorem 2.1 we have $\Gamma \cong \operatorname{PCay}(P)$.

A Haar graph is a bi-Cayley graph of the form $\operatorname{BiCay}(G, \emptyset, \emptyset, S)$. The following is an immediate consequence of the last two propositions.

Corollary 4.5 Every Haar graph can be represented as a 2-partite Cayley graph, and every 2-partite Cayley graph PCay $\left(\left\langle\mathcal{S}_{1}, \mathcal{U}, \mathcal{I} \mid \mathcal{R}_{0}, \mathcal{R}_{1}\right\rangle\right)$ with $\mathcal{S}_{1}=\mathcal{U}=\emptyset$ is a Haar graph.

Most of our motivation for introducing partite presentations came from studying vertex-transitive graphs. Our next proposition gives a sufficient condition for $\mathrm{PCay}(P)$ to be vertex-transitive in terms of the 'symmetry' of $\mathcal{C}(P)$. Given two $\mathrm{CW}$ complexes $\mathcal{C}_{i}$ for $i \in \mathbb{Z} / 2 \mathbb{Z}$, recall that a simplicial map $\phi: \mathcal{C}_{0} \rightarrow \mathcal{C}_{1}$ is a continuous map that maps each $n$-simplex to an $n$-simplex for every $n$. For a CW complex $\mathcal{C}$, the group of bijective simplicial maps from $\mathcal{C}$ to itself is denoted by $\operatorname{Aut}(\mathcal{C})$.

Proposition 4.6 Let $P$ be a 2-partite presentation. As above, the two vertices of the presentation complex $\mathcal{C}$ are denoted by $v_{0}$ and $v_{1}$. If there exists a simplicial map $\phi: \mathcal{C} \rightarrow \mathcal{C}$ such that $\phi\left(v_{0}\right)=v_{1}$, then $P$ Cay $(P)$ is vertex-transitive. 
Proof Set $\Gamma:=\operatorname{PCay}(P)$. Lemma 4.3 says that $G_{i}$ acts transitively on $V_{j}$ for $j \in \mathbb{Z} / 2 \mathbb{Z}$. Thus it only remains to find an automorphism which maps a vertex in $V_{0}$ to a vertex in $V_{1}$. We have a covering map $\epsilon: \widehat{\Gamma} \rightarrow \mathcal{C}$, where $\widehat{\Gamma}$ is the universal cover of $\mathcal{C}$ with 1-skeleton $\Gamma$. By the lifting property $\phi \circ \epsilon: \widehat{\Gamma} \rightarrow \mathcal{C}$ lifts to an automorphism $\widehat{\phi} \in \operatorname{Aut}(\widehat{\Gamma})$ such that $\phi \circ \epsilon=\epsilon \circ \widehat{\phi}$. For any $v \in V_{i}$ we have $\epsilon(v)=v_{i}$ by (4). So for $v \in V_{0}$ we have $\epsilon \circ \widehat{\phi}(v)=\phi \circ \epsilon(v)=\phi\left(v_{0}\right)=v_{1}$ giving that $\widehat{\phi}(v) \in V_{1}$. Thus when restricting $\widehat{\phi}$ to the 1 -skeleton, $\Gamma$, we obtain the required automorphism.

We remark that this sufficient condition is not necessary for $\operatorname{PCay}(P)$ to be vertex-transitive. For example, there is never such an automorphism for the partite presentations $\left\langle\{a\},\{\},\{b\} \mid\left\{a^{n}, a b a^{k} b\right\},\left\{a^{n}\right\}\right\rangle$ of Theorem 3.13 unless $k= \pm 1$. However, we know that $P(n, k)$ is vertex-transitive for many other choices of $n$ and $k$ (such as the case of the Petersen graph $n=5, k=2$ ), see [8].

\section{$5 n$-partite presentations for $n>2$}

\subsection{Definition of partite presentations}

In this section, we generalise our notion of partite presentation by allowing for more than two classes of vertices $V_{i}$. This will allow us to describe vertex-transitive graphs such as the Coxeter graph which cannot be expressed as a bi-Cayley graph.

In Definition 3.1 of a 2-partite presentation we did not explicitly talk about the two vertex classes, but they were implicit in that definition: we had two sets of relators $\mathcal{R}_{0}, \mathcal{R}_{1}$, and the definition of $K$ implicitly distinguished our generators into those staying in the same vertex class, namely $\mathcal{S}_{1}$, from those swapping between the two vertex classes, namely $\mathcal{S}_{2}$. The two vertex classes $V_{i}$ were defined a-posteriori, and Corollary 3.6 confirms that the generators gave rise to edges of the partite Cayley graph behaving this way.

The following definition is a direct generalisation of Definition 3.1, although it is formulated a bit differently. We now make the vertex classes more explicit. The main complication arises from the fact that we have to specify, for each generator $s$, which vertex class any edge coloured by $s$ will lead into if it starts at a given vertex class. This information is encoded as a permutation $\phi(s)$ of the set of vertex classes. As before, we distinguish our generators into two subsets $\mathcal{U}$ and $\mathcal{I}$ to allow for 'involutions' that make partite Cayley graphs with odd degrees possible.

We now give the formal definition:

Definition 5.1 A partite presentation $\langle X|\mathcal{U}| \mathcal{I}|\phi| \mathcal{R}\rangle$ consists of the following data:

1. a set of vertex classes $X$;

2. a generator set $\mathcal{S}$, which is partitioned into two sets $\mathcal{U}$ and $\mathcal{I}$; as before, we use $\mathcal{S}$ to define a group $M F_{P}:=\left\langle\mathcal{S} \mid\left\{s^{2} \mid s \in \mathcal{I}\right\}\right\rangle$ (a free product of cyclic groups each of order 2 or $\infty)$;

3. a map $\phi: \mathcal{S} \rightarrow \operatorname{Sym}_{X}$ from the generator set to the group $\operatorname{Sym}_{X}$ of permutations of $X$; We remark that any such map defines an action of $M F_{P}$ on $X$ via $s_{1} \ldots s_{n}$. $x:=\phi\left(s_{1}\right) \circ \ldots \circ \phi\left(s_{n}\right)(x)$, where $s_{i} \in \mathcal{S} \cup \mathcal{S}^{-1}$, and $\phi\left(s^{-1}\right):=\phi(s)^{-1}$. We require that 
(a) this action of $M F_{P}$ on $X$ is transitive, and

(b) for all $s \in \mathcal{I}$ the permutation $\phi(s)$ is fixed point free of order 2;

4. a relator set $\mathcal{R}_{x} \subset \operatorname{Stab}\left(M F_{P}, x\right)=$ for each $x \in X$, where $\operatorname{Stab}\left(M F_{P}, x\right)$ denotes the stabiliser of $x$ with respect to the aforementioned action of $M F_{P}$. (This is a natural condition, as we want to return to our starting vertex when following a walk labelled by a relator, and in particular we want to return to the same vertex class.)

The set $\left\{\mathcal{R}_{x}: x \in X\right\}$ of these relator sets is denoted by $\mathcal{R}$.

We now use such a presentation $P=\langle X|\mathcal{U}| \mathcal{I}|\phi| \mathcal{R}\rangle$ to define the partite Cayley graph PCay $(P)$, in analogy with Definition 3.7. We start by defining the presentation graph $C(P)$. This has vertex set $X$, and directed edge set $\{(x, \phi(s)(x) \mid$ for all $x \in X$ and $\left.s \in \mathcal{U} \cup(\mathcal{U})^{-1} \cup \mathcal{I}\right\}$ where $\phi\left(s^{-1}\right)=\phi(s)^{-1}$. We colour it by $c: \vec{E}(C(P)) \rightarrow$ $\mathcal{S} \cup \mathcal{S}^{-1}$ defined by $c(x, \phi(s) x):=s$, and note that this is a Cayley-like colouring as in Definition 3.8.

The partite presentation complex $\mathcal{C}(P)$ is the 2-complex obtained from $C(P)$ as follows. For each $x \in X$ and each $r \in \mathcal{R}_{x}$, we introducing a 2-cell and glue its boundary along the walk of $C(P)$ starting at $x$ and dictated by $r$ (as in Definition 3.9). It is straightforward to check that this is a closed walk using (4).

Note that $\mathcal{C}(P)$ is connected by condition (a) Finally,

Definition 5.2 We define the partite Cayley graph $\operatorname{PCay}(P)=\operatorname{PCay}\langle X|\mathcal{U}| \mathcal{I}|\phi| \mathcal{R}\rangle$ to be the 1-skeleton of the universal cover of $\mathcal{C}(P)$.

Letting $\epsilon: \mathrm{PCay}(P) \rightarrow C(P)$ be the covering map, we can lift $c$ to the edgecolouring $\tilde{c}=c \circ \epsilon$ of $\operatorname{PCay}(P)$.

Note that if $X$ is a singleton, then we recover the usual group presentations and Cayley graphs by the above definitions. Our 2-partite presentations $\left\langle\mathcal{S}_{1}, \mathcal{U}^{\prime}, \mathcal{I}^{\prime} \mid \mathcal{R}_{0}, \mathcal{R}_{1}\right\rangle$ of Sect. 3 are tantamount to partite presentations as in Definition 5.1 with $X=\{0,1\}$, where $\phi\left(s_{1}\right)=(0)(1)$ for $s_{1} \in \mathcal{S}_{1}$ and $\phi\left(s_{2}\right)=(0,1)$ for $s_{2} \in \mathcal{S}_{2}:=\mathcal{U}^{\prime} \cup \mathcal{I}^{\prime}$, with $\mathcal{U}=\mathcal{S}_{1} \cup \mathcal{U}^{\prime}$ and $\mathcal{I}=\mathcal{I}^{\prime}$.

As in Sect. 3, we can alternatively define $\operatorname{PCay}(P)$ as a graph quotient, following the lines of Definition 3.2, as follows:

1. Let $\mathcal{S}:=\mathcal{U} \cup \mathcal{I}$ and define the group $M F_{P}$ by the presentation $\left\langle\mathcal{S} \mid\left\{s^{2}: s \in \mathcal{I}\right\}\right\rangle$; this is a free product of infinite cyclic groups, one for each $s \in \mathcal{U}$, and cyclic groups of order 2 , one for each $s \in \mathcal{I}$. Define the tree $T_{P}$ by

$$
\begin{aligned}
V\left(T_{P}\right) & :=M F_{P}, \text { and } \\
\vec{E}\left(T_{P}\right) & :=\left\{(w, w s) \mid w \in M F_{P}, s \in \mathcal{S} \cup \mathcal{S}^{-1}\right\} .
\end{aligned}
$$

This is a $(2|\mathcal{U}|+|\mathcal{I}|)$-regular tree, and it comes with a colouring $c: \vec{E}\left(T_{P}\right) \rightarrow$ $\mathcal{S} \cup \mathcal{S}^{-1}$ by $c(w, w s)=s$.

2. We can extend the map $\phi$ of (3) from $\mathcal{S}$ to an action of $M F_{P}$ by composition: we let $x \cdot s_{1} \ldots s_{n}:=\phi\left(s_{n}\right) \circ \ldots \circ \phi\left(s_{1}\right)(x)$ for all $x \in X$ and $s_{i} \in \mathcal{S}$. Let $W_{x, y}=\{w \in$ $\left.M F_{P} \mid \phi(w)(x)=y\right\}$ for $x, y \in X$. Fixing any 'base' vertex class $b \in X$ leads 
to a partition of $V\left(T_{P}\right)=M F_{P}$, namely $\tilde{V}_{x}=W_{b, x}$. Note that two vertices in $u, v \in \tilde{V}_{x} \subset M F_{P}$ differ by a word $u^{-1} v \in W_{x, x}=\operatorname{Stab}\left(M F_{P}, x\right)$.

3. Let $R_{x}=\left\langle w r w^{-1} \mid r \in \mathcal{R}_{y}, w \in W_{x, y}, y \in X\right\rangle \subset W_{x, x}$. Then we say that two vertices in $u, v \in \tilde{V}_{x}$ are equivalent, and write $u \sim v$, if $u^{-1} v \in R_{x}$. Similarly, for edges $e, f \in \vec{E}\left(T_{P}\right)$ we write $e \sim f$ if $c(e)=c(f)$ and $\tau(e) \sim \tau(f)$ and $\tau\left(e^{-1}\right) \sim \tau\left(f^{-1}\right)$.

4. We define $\operatorname{PCay}(P)$ to be the corresponding quotient $T_{P} / \sim$.

As in Corollary 3.6, it is not hard to see that $T_{P}$ is the universal cover of PCay $(P)$. Define $V_{x}, x \in X$ as the image of $\tilde{V}_{x}$ under the quotient of $\sim$. We have $W_{x, x}=\pi_{1}(C(P), x)$ and $\pi_{1}(\mathcal{C}(P), x)=R_{x} \backslash W_{x, x}=: G_{x}$, analogously to the 2-partite presentation case. We call $G_{x}, x \in X$ the vertex groups.

We remark that the vertex set of $\operatorname{PCay}(P)$ can be given the structure of a groupoid $\mathcal{G}_{\text {PCay }(P)}$. Indeed, we can think of $\bigcup_{x, y \in X} W_{x, y}$ as the ground set, and define the groupoid operation $W_{x, y} \times W_{y, z} \rightarrow W_{x, z}$ by concatenation. Another way to think of this groupoid is $\mathcal{G}_{\mathrm{PCay}(P)} \cong \pi_{1}(\mathcal{C}(P), X)$, the universal groupoid of the presentation complex $\mathcal{C}(P)$, with paths starting and ending in $V(C)$.

The main result of this section is that every vertex-transitive graph $\Gamma$ is isomorphic to $\operatorname{PCay}(P)$ for some partite presentation $P$. For the proof of this we will need to decompose the edges of $\Gamma$ into cycles. The next section discusses such decompositions.

\subsection{Multicycle colourings}

Leighton [17] asked whether finite vertex-transitive graphs have similar colouring structures to Cayley graphs of groups. For a Cayley graph $\Gamma=\operatorname{Cay}(G, \mathcal{S})$, the generators canonically induce a colouring $c: E(\Gamma) \rightarrow \mathcal{S}$ as above, so that $c^{-1}(s)$ is a disjoint union of cycles of the same length for every $s \in \mathcal{S}$. In the finite case Leighton called this a multicycle. A double-ray is a 2-way infinite path.

Definition 5.3 A graph $\Delta$ is said to be a multicycle, if either every component of $\Delta$ is a cycle of a fixed length, or every component of $\Delta$ is a double-ray, or every component of $\Delta$ is an edge. A multicycle colouring of a graph $\Gamma$ is a colouring $c: E(\Gamma) \rightarrow \Omega$ such that the graph with vertex set $V(\Gamma)$ and edge set $c^{-1}(x)$ is a multicycle for each $x \in \Omega$.

Thus every Cayley graph has a multicycle colouring, namely its natural colouring by the generators. Leighton [17] conjectured that every finite vertex-transitive graph has a multicycle colouring [17], but this was shown to be false by Marušič [20], a counter-example being the line graph of the Petersen graph:

Example 5.4 Given a graph $\Delta$ we construct the line graph $\Gamma:=L(\Delta)$ as follows. We set $V(\Gamma):=E(\Delta)$ and $\vec{E}(\Gamma)=\left\{\left(e, e^{\prime}\right) \mid \tau\left(e^{ \pm 1}\right)=\tau\left(e^{ \pm 1}\right)\right\}$. To see there is no multicycle colouring of $L(P(5,2))$, note that it has $|V(L(P(5,2)))|=|E(P(5,2))|=15$ vertices, so any multicycle will have to consist of triangles, pentagons, or 15-cycles. Any 15 -cycle in $L(P(5,2))$ would yield a Hamiltonian cycle in $P(5,2)$, which we know does not exist. Moreover, the only triangles in $L(P(5,2))$ are formed by edges incident with a single vertex of $P(5,2)$. As $P(5,2)$ is not bipartite, there is no way to 
Fig. 4 The line graph $L(P(5,2))$ of the Petersen graph

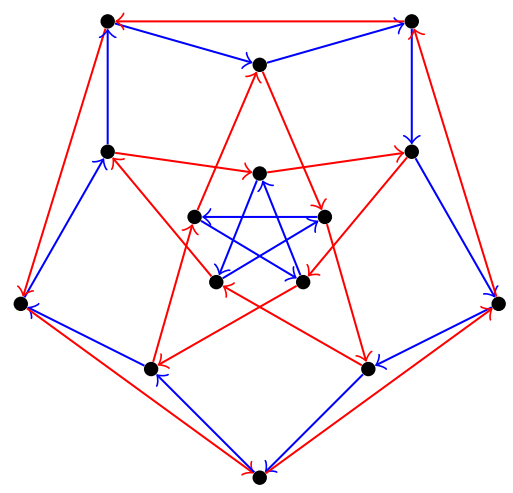

partition the triangles into disjoint sets that pass through all vertices. So we can only use sets of five cycles, which correspond to sets of edge disjoint pentagons in $P(5,2)$. As $P(5,2)$ is cubic, there is no set of pentagons that visits every edge exactly once.

Still, it is possible to express $L(P(5,2))$ as a partite Cayley graph: $\mathrm{PCay}\left\langle a \mapsto(12)(3), b \mapsto(1)(23) \mid\left\{b^{5}, a^{10}, a^{2} b\right\},\left\{a^{-2} b^{4}\right\},\left\{a^{5}, b^{10}, b^{2} a\right\}\right\rangle$

Our aim now is to weaken the notion of a multicycle colouring enough that every vertex-transitive graph will admit one, so that the weakened notion will allow us to find partite presentations. This is the essence of Theorem 5.7.

Definition 5.5 A graph $\Gamma$ is a weak multicycle, if it is a vertex-disjoint union of cycles, double-rays, and edges. A weak multicycle colouring of a graph $\Gamma$ is a colouring $c: E(\Gamma) \rightarrow \Omega$ such that the graph with vertex set $V(\Gamma)$ and edge set $c^{-1}(x)$ is a weak multicycle for each $x \in \Omega$.

We say that a weak multicycle colouring $c$ is partition-friendly, if $c^{-1}(x)$ is regular for all $x \in \Omega$. In other words, $c^{-1}(x)$ is either a disjoint union of cycles or a perfect matching for all $x$.

As we will see in the following section, every vertex-transitive graph has a partitionfriendly weak multicycle colouring. The condition of vertex transitivity here cannot be relaxed to just regularity. Indeed, let $\Gamma$ be the 3-regular graph in Fig. 5. Since its vertex degrees are odd, one of the colours in any weak multicycle colouring must induce a perfect matching. But $\Gamma$ does not have a perfect matching $M$, because removing $v$ and the vertex matched to $v$ by $M$ results in at least one component with an odd number of vertices.

\subsection{Multicycle colourings and partite presentations}

We say a partite presentation $P=\langle X|\mathcal{U}| \mathcal{I}|\phi| \mathcal{R}\rangle$ is uniform, if for every $s \in \mathcal{S}$, all orbits of $\phi(s)$ have the same size. In other words, if $c$ is a multicycle colouring on $C(P)$. In light of Leighton's aforementioned conjecture, one can ask the following:

Question 5.6 Let $\Gamma$ be a vertex-transitive graph. Does $\Gamma$ have a multi-cycle colouring if and only if it is the partite Cayley graph of a uniform partite presentation? 
Fig. 5 A regular graph with no partition-friendly weak multicycle colouring
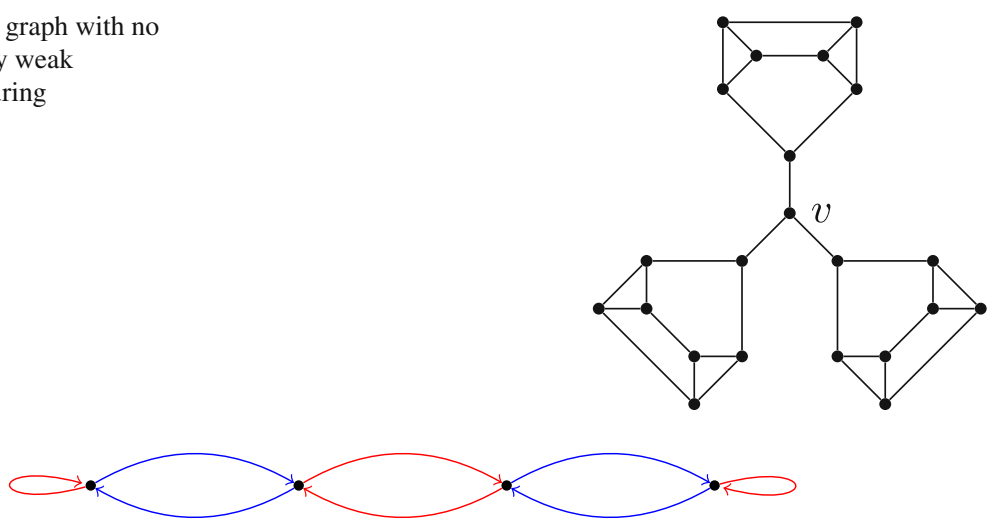

Fig. 6 PCay $\left\langle\{a\},\{b\}, \varnothing \mid\{a\},\left\{a^{2}\right\}\right\rangle$

The forward direction is true: if $\Gamma$ has a multicycle colouring then it has a uniform partite presentation given in the proof of Theorem 5.7. But the backward direction is false, as shown by the following example. Consider the 2-partite presentation $P=\left\langle\{a\},\{b\}, \emptyset \mid\{a\},\left\{a^{2}\right\}\right\rangle$. This is trivially uniform, like every 2-partite presentation. However, PCay $(P)$, shown in Fig. 6, does not have a multicycle colouring.

The following result will be used later to show that every vertex-transitive graph admits a partite presentation.

Theorem 5.7 A connected graph has a partition-friendly weak multicycle colouring if and only if it admits a partite presentation.

Proof Recall that a graph is defined using a directed edge set $\vec{E}(\Gamma)$, but we can also consider the undirected edge set $E(\Gamma)=\vec{E}(\Gamma) /^{-1}$, so that an undirected edge is a pair $\{e, d\}$ such that $e^{-1}=d$ and $d^{-1}=e$. In the following proof we have to transition between colourings of the directed edges and colourings of the undirected edges. Apart from this, the proof boils down to a straightforward checking of the conditions of the corresponding definitions.

For the forward direction, suppose $\Gamma$ is connected and it has a partition-friendly weak multicycle colouring $c: E(\Gamma) \rightarrow \Omega$. To define the desired partite presentation $P$, we start with

- $X=V(\Gamma)$,

- $\mathcal{U}=\left\{\omega \in \Omega \mid c^{-1}(\omega)\right.$ is of degree 2$\}$, and

- $\mathcal{I}=\left\{\omega \in \Omega \mid c^{-1}(\omega)\right.$ is of degree 1$\}$.

Since $c$ is partition-friendly, we have $\mathcal{U} \cup \mathcal{I}=\Omega$. We want to refine $c$ into a colouring $c^{\prime}$ of the directed edges of $\Gamma$. To do this, for each $\omega \in \mathcal{U}$ we choose an orientation $O_{\omega} \subset \vec{E}(\Gamma)$ of $\left.c^{-1}(\omega)\right\} \subset E(\Gamma)$ (recall this means that $\left(O_{\omega} \cup O_{\omega}^{-1}\right) /^{-1}=c^{-1}(\omega)$ and $\left.O_{\omega} \cap O_{\omega}^{-1}=\emptyset\right)$. Since $c^{-1}(\omega)$ is a multicycle, we can choose $O_{\omega}$ so that each of its cycles is oriented, that is, for each vertex $v \in V(\Gamma)$ there is exactly one $e \in$ $O_{\omega}$ with $\tau(e)=x$. Thus $O_{\omega}$ defines a permutation $\phi(\omega)$ of $X=V(\Gamma)$, by letting $\phi(\omega)(x)$ be the unique $y \in X$ such that $(x, y) \in O_{\omega}$. Moreover, for each $\omega \in \mathcal{I}$, let 
$O_{\omega}=\left\{e \in \vec{E}(\Gamma) \mid[e] \in c^{-1}(\omega)\right\}$, and let $\phi(\omega)$ be the involution of $V(\Gamma)$ exchanging the end-vertices of each edge in $c^{-1}(\omega)$. Thus $\phi$ satisfies (b) of Definition 5.1 by construction (we will check (a) below).

We now define $c^{\prime}$ by

$$
c^{\prime}(e)= \begin{cases}c([e]) & \text { if } e \in O_{c([e])} \\ c([e])^{-1} & \text { otherwise. }\end{cases}
$$

This maps $E(\Gamma)$ to $\mathcal{U} \cup \mathcal{U}^{-1} \cup \mathcal{I}$, because for $e \in \vec{E}(\Gamma)$ such that $c([e]) \in \mathcal{I}$ we have $e, e^{-1} \in O_{c\left(\left[e^{-1}\right]\right)}$ by definition. Easily, $c^{\prime}$ is a Cayley-like colouring. This allows us to define $\mathcal{W}_{v}$ on $\Gamma$ as described after Definition 3.8. Note that as $\Gamma$ is connected, for any two $x, y \in V(\Gamma)$ there is a path $p$ connecting $x$ and $y$. Then the path $p$ corresponds to a word $\mathcal{W}_{x}(p) \in M F_{P}$ such that $\phi\left(\mathcal{W}_{x}(p)\right)(x)=y$. Therefore, the action of $M F_{P}$ on $X=V(\Gamma)$ is transitive as required by (a) of Definition 5.1.

To complete the definition of our partite presentation $P$, we choose the relators

- $\mathcal{R}_{v}=\mathcal{W}_{v}\left(\pi_{1}(\Gamma, v)\right) \subset M F_{P}$.

We claim that $\Gamma$ coincides with the presentation graph $C(P)$. To begin with, they have the same vertex set $V(C)=X=V(\Gamma)$. Moreover,

$$
\begin{aligned}
\vec{E}(C(P)) & =\left\{(x, \phi(\omega)(x)) \mid x \in V(\Gamma), \omega \in \mathcal{U} \cup \mathcal{U}^{-1} \cup \mathcal{I}\right\} \\
& =\cup_{\omega \in \Omega}\left\{(x, y) \mid(x, y) \in O_{\omega} \text { or }(y, x) \in O_{\omega}\right\} \\
& =\cup_{\omega \in \Omega}\left(c^{\prime}\right)^{-1}(\omega)=\vec{E}(\Gamma)
\end{aligned}
$$

and so our claim is proved.

As we defined $\mathcal{C}(P)$ by glueing in a 2-cell along each closed walk dictated by an element of $\mathcal{R}_{v}, v \in V(C(P))$, where we have chosen $\mathcal{R}_{v}=\mathcal{W}_{v}\left(\pi_{1}(\Gamma, v)\right)$, we have forced $\pi_{1}(\mathcal{C}(P), v)$ to be trivial. Therefore, $\mathcal{C}(P)$ coincides with its own universal cover $\widehat{\mathcal{C}(P)}$. Thus PCay $(P)$, defined as the 1 -skeleton of $\widehat{\mathcal{C}(P)}$, is $C(P)=\Gamma$. Therefore, $P$ is a partite presentation for $\Gamma$.

For the converse direction, let $\Gamma=\operatorname{PCay}(P)$ for some partite presentation $P$. Let $\epsilon: \Gamma \rightarrow C(P)$ be the covering map, and $c_{C}^{\prime}: \vec{E}(C) \rightarrow \mathcal{U} \cup \mathcal{U}^{-1} \cup \mathcal{I}$ the colouring induced by the generators of $P$, as in the definition of PCay $(P)$. We collapse $c_{C}^{\prime}$ into a colouring $c_{C}$ of the undirected edges of $C$ defined by

$$
c_{C}([e])= \begin{cases}u \in \mathcal{U} & \text { if } c(e) \in\left\{u, u^{-1}\right\} \\ i \in \mathcal{I} & \text { if } c(e)=i .\end{cases}
$$

We can collapse $c_{\Gamma}^{\prime}: \vec{E}(\Gamma) \rightarrow \mathcal{U} \cup \mathcal{U}^{-1} \cup \mathcal{I}$ similarly to obtain an undirected colouring $c_{\Gamma}: E(\Gamma) \rightarrow \mathcal{U} \cup \mathcal{I}$. Note that $c_{C}$ is a partition-friendly weak multicycle colouring, with $c^{-1}(i)$ being of degree 1 for $i \in \mathcal{I}$ and $c^{-1}(u)$ being of degree 2 for $u \in \mathcal{U}$, by the definitions. As $c_{C}^{\prime} \circ \epsilon=c_{\Gamma}^{\prime}$, it is easy to verify that $c_{C} \circ \epsilon=c_{\Gamma}$. This implies that $c_{\Gamma}^{-1}(x)$ has the same degree as $c_{C}^{-1}(x)$, and that every vertex has 
at least one incident edge coloured $s$ for each $s \in \mathcal{I} \cup \mathcal{U}$. This means that $c_{\Gamma}$ is a partition-friendly weak multicycle colouring of $\Gamma$ as claimed.

\subsection{Weak multicycle colourings of vertex-transitive graphs}

The aim of this section is to show that every vertex-transitive graph $\Gamma$ has a partition-friendly weak multicycle colouring; hence it admits a partite presentation by Theorem 5.7 .

For this, we will use the following result of Godsil and Royle [11, Theorem 3.5.1]:

Theorem 5.8 (Godsil \& Royle [11, Theorem 3.5.1]) Let $\Gamma$ be a connected, finite, vertex-transitive graph. Then $\Gamma$ has a matching that misses at most one vertex.

This result generalises to infinite vertex-transitive graphs as follows:

Theorem 5.9 ([3,16]) Let $\Gamma$ be a countably infinite, connected, vertex-transitive graph. Then $\Gamma$ has a perfect matching.

The proof of Theorem 5.9 in the locally finite case can be found in [3] or [16, Proposition 3.2.17] ${ }^{1}$ If $\Gamma$ is not locally finite, then it is easy to construct a perfect matching greedily.

In passing, let us mention the following still open conjecture. If true, it would imply that all finite vertex-transitive cubic graphs have a uniform partite presentation.

Conjecture 5.10 (Lovasz [18, Problem 11]) Let $\Gamma$ be a finite cubic vertex-transitive graph. Then there exists a perfect matching $M$ in $\Gamma$ such that $\Gamma \backslash M$ consists of either one cycle, (and $\Gamma$ is Hamiltonian), or of two disjoint cycles of the same length.

The following old theorem of Petersen is a rather straightforward application of Hall's Marriage theorem [12]. Although it is well-known, we include a proof for convenience.

Theorem 5.11 (J. Petersen [23]) Every regular graph of positive (finite and) even degree has a spanning 2-regular subgraph.

Proof Let $\Gamma$ be a $2 k$-regular graph. If $\Gamma$ is finite then it contains an Euler tour $C$ (i.e. a closed walk that uses each edge exactly once) by Euler's theorem [5]. Pick an orientation of $O_{C} \subset \vec{E}(\Gamma)$ of $C$. If $\Gamma$ is infinite then just choose an orientation with equal in and out degree, which can be constructed greedily. Then construct an auxiliary graph $\Delta$ with

$$
\begin{aligned}
& V(\Delta)=\left\{v^{+}, v^{i} \mid v \in V(\Gamma)\right\}, \text { and } \\
& E(\Delta)=\left\{\left(v^{+}, u^{i}\right) \mid(v, u) \in O_{C}\right\}
\end{aligned}
$$

By definition, $\Delta$ is $k$-regular and bipartite, with bipartition $V^{+}=\left\{v^{+} \mid v \in V(\Gamma)\right\}$ and $V^{-}=\left\{v^{-} \mid v \in V(\Gamma)\right\}$. For any finite $A \subset V^{+}$, as $\Delta$ is $k$-regular, the neighbourhood

\footnotetext{
${ }^{1}$ Leemann [16] states this for graphs of odd degree only, but the proof applies as is to the even degree case. An alternative proof can be found in Appendix of https: / /arxiv.org/abs/2007.06432v1.
} 
$N(A)=\left\{u^{-} \mid\left(v^{+}, u^{-}\right) \in E(\Delta)\right.$ with $\left.v^{+} \in A\right\}$ of $A$ has size at least $k \times|A| / k=|A|$. So by Hall's Marriage theorem [12], $\Delta$ contains a perfect matching $M \subset E(\Delta)$. Then the spanning subgraph $S \subset \Gamma$ given by $\left\{(v, u) \mid\left(v^{+}, u^{-}\right) \in M\right\} \subset E(\Gamma)$ is 2-regular by construction.

Combining this with Theorem 5.8 and Theorem 5.9, we now obtain

Lemma 5.12 Every countable, vertex-transitive, graph $\Gamma$ has a partition-friendly weak multicycle colouring.

Proof We first consider the case where $\Gamma$ is (finite or) locally finite. As $\Gamma$ is vertextransitive it is $n$-regular for some $n \in \mathbb{N}$. If $n$ is even, then we can apply Theorem 5.11 recursively to decompose $E(\Gamma)$ into 2-regular spanning subgraphs, and attributing a distinct colour to the edges of each of those subgraphs yields a partition-friendly weak multicycle colouring.

If $n$ is odd, then we first find a perfect matching $M$, colour its edges with the same colour, and treat $\Gamma \backslash M$ as above to obtain a partition-friendly weak multicycle colouring. To obtain $M$, note that if $\Gamma$ is finite, then $|V(G)|$ is even since $|E(\Gamma)|=$ $n|V(G)| / 2$. Therefore, $\Gamma$ has a perfect matching by Theorem 5.8 as no matching can miss exactly 1 vertex in this case. If $\Gamma$ is infinite, then Theorem 5.9 provides a perfect matching.

If $\Gamma$ is not locally finite, then each vertex has countably infinite degree. We will decompose $E(\Gamma)$ into an edge-disjoint union of multicycles $\left\{M_{i}\right\}_{i \in \mathbb{N}}$, where each $M_{i}$ is a spanning subgraph consisting of pairwise vertex-disjoint double-rays. For this, let $\left\{e_{i}\right\}_{i \in \mathbb{N}}$ be an enumeration of the edges of $\Gamma$, and let $\left\{v_{i}\right\}_{i \in \mathbb{N}}$ be a sequence of vertices of $\Gamma$ in which each $v \in V(\Gamma)$ appears infinitely often. We greedily construct an $M_{0}$ as above containing $e_{0}$ as follows. We start with $M_{0}^{0}=e_{0}$, and for $i=1,2, \ldots$, we extend the (possibly trivial) path in $M_{0}^{i-1}$ containing $v_{i}$ into a longer path by adding an edge of $\Gamma-M_{0}^{i-1}$ at each of its end-vertices. As $M_{0}^{i-1}$ is finite, and every vertex has infinite degree, this is always possible. Finally, we let $M_{0}:=\bigcup_{i \in \mathbb{N}} M_{0}^{i}$. Since each $v \in V(\Gamma)$ appears infinitely often as $v_{i}$, we deduce that $M_{0}$ is a spanning union of vertex-disjoint double-rays as desired.

Having constructed $M_{0}$, we inductively construct the $M_{i}, i \geq 1$ so that $M_{i}$ contains $e_{i}$ unless $e_{i}$ is already in $\bigcup_{j<i} E\left(M_{j}\right)$, by noticing that $\Gamma-\bigcup_{j<i} E\left(M_{j}\right)$ is a regular graph with countably infinite degree itself, and repeating the above procedure. Then $\left\{M_{i}\right\}_{i \in \mathbb{N}}$ is the desired partition-friendly weak multicycle colouring of $\Gamma$.

This combined with Theorem 5.7 yields our main result:

Theorem 1.1 Every countable, vertex-transitive, graph has a partite presentation.

We conclude this section with the following question.

Question 5.13 For a vertex-transitive graph $\Gamma$ with partite presentation $P$ does

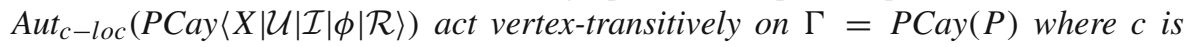
the colouring coming from $P$ ? 


\subsection{Generalised results}

Here we extend some of our earlier results from 2-partite to general partite presentations. Where the same arguments apply directly the proofs will be omitted. First we generalise Lemma 4.3:

Proposition 5.14 For a partite presentation $P=\langle X|\mathcal{U}| \mathcal{I}|\phi| \mathcal{R}\rangle$ there is a natural inclusion of the vertex group $G_{x} \leq A u t_{c}(P C a y(P))$ for each $x \in X$. Moreover, $G_{x}$ acts regularly on $V_{x}$, and so $P C a y(P)$ is $|X|$-Cayley.

The vertex groups are still isomorphic due to the fact that $\pi_{1}$ does not depend on the choice of a base point:

Proposition 5.15 For every partite presentation $P=\langle X|\mathcal{U}| \mathcal{I}|\phi| \mathcal{R}\rangle$, and every $x, y \in$ $X$, the vertex groups $G_{x}, G_{y}$ are isomorphic.

Proof As above, let $C(P)=: C$ be the presentation graph of $P$. Let $x, y \in X=V(C)$. Recall that $G_{x}:=W_{x, x} / R_{x}$ is the right quotient of $W_{x, x}$ by $R_{x}$, where $W_{x, z}$ is the set of paths in $C$ from $x$ to $z$ up to homotopy (in particular, $W_{x, x}=\pi_{1}(C, x)$ ), and

$$
R_{x}:=\left\langle\left\{w \mathcal{W}_{z}^{-1}(r) w^{-1} \mid r \in \mathcal{R}_{z}, w \in W_{x, z}, z \in X\right\}\right\rangle
$$

with $\mathcal{W}_{z}^{-1}$ the map from words in $M F_{P}$ to paths in $C$ defined in Sect. 2. Let $p \in W_{x, y}$ be a path from $x$ to $y$ in $C$. As $\pi_{1}$ is base point preserving, we have

$$
W_{x, x}=p W_{y, y} p^{-1} \text {. }
$$

Moreover, note that

$$
\begin{aligned}
p R_{y} p^{-1} & =\left\langle\left\{(p w) \mathcal{W}_{z}^{-1}(r)(p w)^{-1} \mid r \in \mathcal{R}_{z}, w \in W_{y, z}, z \in X\right\}\right\rangle \\
& =\left\langle\left\{w^{\prime} \mathcal{W}_{z}^{-1}(r)\left(w^{\prime}\right)^{-1} \mid r \in \mathcal{R}_{z}, w^{\prime} \in W_{x, z}, z \in X\right\}\right\rangle \\
& =R_{x} .
\end{aligned}
$$

This defines a homomorphism $\phi: G_{y} \rightarrow G_{x}$ by $\phi: w R_{y} \mapsto p w p^{-1} R_{x}$ for every $w \in W_{y, y}$. It is surjective by (7) and injective as $p R_{y} p^{-1}=R_{x}$. Thus it is an isomorphism proving our claim.

We generalise Proposition 4.6 to obtain a sufficient condition for vertex transitivity.

Proposition 5.16 Let $P=\langle X|\mathcal{U}| \mathcal{I}|\phi| \mathcal{R}\rangle$ be a partite presentation. If the presentation complex $\mathcal{C}(P)$ is vertex-transitive, then so is $P \operatorname{Cay}(P)$.

\subsection{Quasi-isometry to vertex groups}

It is a straightforward consequence of the Švarc-Milnor lemma [21] that if $P=$ $\langle X|\mathcal{U}| \mathcal{I}|\phi| \mathcal{R}\rangle$ is a partite presentation with finite $X$, then $\Gamma:=\operatorname{PCay}(P)$ is quasiisometric to (any Cayley graph of) $G_{x}$. In this section, we provide the details for 
the non-expert reader. This will be used in Sect. 7 to argue that there are partite Cayley graphs that cannot be represented by a partite presentation with finite $X$.

A quasi-isometry between metric spaces $(X, d)$ and $\left(Y, d^{\prime}\right)$ is a (not necessarily continuous) function $f: X \rightarrow Y$ satisfying the following two statements for some constants $A, B \in \mathbb{R}_{+}$:

$$
\frac{1}{A} d(x, z)-B \leq d^{\prime}(f(x), f(z) \leq A d(x, z)+B
$$

for every $x, z \in X$, and for every $y \in Y$ there is $x \in X$ such that $d^{\prime}(f(x), y) \leq B$.

If such an $f$ exists, we say that $(X, d)$ and $\left(Y, d^{\prime}\right)$ are quasi-isometric to each other. (Easily, this is an equivalence relation.) It is well-known, and easy to check, that any two finitely generated Cayley graphs of the same group are quasi-isometric to each other. We say that a metric space $(X, d)$ is quasi-isometric to a group $G$, if $(X, d)$ is quasi-isometric to some, hence to every, finitely generated Cayley graph of $G$.

Proposition 5.17 Let $P=\langle X|\mathcal{U}| \mathcal{I}|\phi| \mathcal{R}\rangle$ be a partite presentation with finite $X$. Then $\Gamma:=P \operatorname{Cay}(P)$ is quasi-isometric to $G_{x}$ for every $x \in X$.

Proof Consider the inclusion map $i: C \rightarrow \mathcal{C}$ from the presentation graph $C:=C(P)$ to the presentation complex $\mathcal{C}:=\mathcal{C}(P)$ of $P$. It is well-known [13, Proposition 1.26] that the inclusion of the one skeleton into a 2-complex induces a surjection on the level of fundamental groups, and the kernel is exactly the normal closure of the words bounding the 2-cells. Thus $i_{*}: \pi_{1}(C, x) \rightarrow \pi_{1}(\mathcal{C}, x)$ is a surjection with kernel $R_{x}$, so that $\pi_{1}(\mathcal{C}, x)=\pi_{1}(C, x) / R_{x}=W_{x, x} / R_{x}=G_{x}$.

Let $\widehat{\Gamma}$ be the universal cover of $\mathcal{C}$, with covering map $\widehat{\eta}: \widehat{\Gamma} \rightarrow \mathcal{C}$. As $\pi_{1}(\mathcal{C}, x)=$ $G_{x}$ we have an action of $G_{x}$ on $\widehat{\Gamma}$-and it's 1-skeleton $\operatorname{PCay}(P)=: \Gamma-$ by deck transformations. We know the quotient of a universal cover by the group of deck transformations is the space itself [13, p 70]. Thus the quotient of $\widehat{\Gamma}$ by $G_{x}$ is $\mathcal{C}$, and so the quotient of $\Gamma$ by $G_{x}$ is $C$. Since $C$ is finite when $X$ is, we deduce that the action of $G_{x}$ on $\Gamma$ is co-compact.

Lastly, we claim that the action of $G_{x}$ by deck transformations on $\Gamma$ is properly discontinuous. Any compact subset $K \subset \Gamma$ is bounded in the graph metric. By Proposition 5.14, $G_{x}$ acts regularly on $V_{x}$, and in particular the stabiliser of each vertex is trivial. Our claim now easily follows, e.g. by using the fact that every cellular action on a CW-complex with finite stabilisers of cells is properly discontinuous $[15$, Theorem $9,(2)=(10)]$.

To summarise, the action of $G_{x}$ on $\Gamma$ is properly discontinuous and co-compact. The Švarc-Milnor lemma [21] says exactly that $G_{x}$ is finitely generated, and quasiisometric to $\Gamma$ for any such action.

\section{Line graphs of Cayley graphs admit partite presentations}

In this section, we show that every line graph of a Cayley graph can be represented as a partite Cayley graph. For this we will use 1- and 2-factorisations of the complete graphs as a tool. Let $K_{n}$ be the complete graph on $n$-vertices. If $n$ is odd then $K_{n}$ has 
a Hamiltonian decomposition, a partition of the edges into spanning cycles [1]. If $n$ is even, then a special case of Baranyai's theorem [2] gives us a 1-factorisation of $K_{n}$, i.e. a partition of the edges into perfect matchings.

Thus in either case, we have found a partition-friendly multicycle colouring $c$ : $E\left(K_{n}\right) \rightarrow \Omega$ of $K_{n}$. Next, we want to associate each colour $\omega \in \Omega$ with a permutation $\pi_{\omega} \in \operatorname{Sym}_{n}$ of the vertices of $K_{n}$. To do so, for each $\omega \in \Omega$ such that $c^{-1}(\omega)$ is 2-regular, we pick an orientation $O_{\omega} \subset c^{-1}(\omega)$, (such that $O_{\omega} \cap O_{\omega}^{-1}=\varnothing$ and $O_{\omega} \cup O_{\omega}^{-1}=c^{-1}(\omega)$ ), and let $\pi_{\omega}$ be the corresponding permutation (sending each vertex to its successor in $O_{\omega}$. For each $\omega \in \Omega$ such that $c^{-1}(\omega)$ is 1 -regular, we let $\pi_{\omega}$ be the permutation that exchanges the two end-vertices of each edge in $c^{-1}(\omega)$.

Proposition 6.1 Let $\Gamma=\operatorname{Cay}\langle\mathcal{S} \mid \mathcal{R}\rangle$ be a Cayley graph. Then the line graph $L(\Gamma)$ can be represented as $P C a y(P)$ for a partite presentation $P$ with at most $|\mathcal{S}|$ vertex classes.

Proof The partite presentation $P$ we will construct will have one vertex class for each generator in $\mathcal{S}$. Since the edges of $L(\Gamma)$ are precisely the pairs of incident edges of $\Gamma$, we will identify the generators of $P$ with pairs of generators $s, t \in \mathcal{S}$. Since we need to pay attention to the directions of the edges of $\Gamma$, each such pair $s, t$ will give rise to four generators of $P$, indexed by the elements of $\{-1,1\}^{2}$. Similarly, each $s \in \mathcal{S}$ will give rise to two generators of $P$, since there are pairs of incident edges of $\Gamma$ labelled by $s$, and there are two choices for their directions. The relators of $P$ will be of two kinds. The first kind is just obtained by rewriting the elements of $\mathcal{R}$ in terms of the new generators. The second kind will correspond to closed walks in $L(\Gamma)$ contained in the star of a vertex of $\Gamma$.

We proceed with the formal definition of $P$. The vertex classes of $P$ will be identified with the generating set $\mathcal{S}$ of $\Gamma$. Let $K_{\mathcal{S}}$ denote the complete graph with $V\left(K_{\mathcal{S}}\right)=\mathcal{S}$. From the above discussion we obtain a multicycle colouring $M \subset \operatorname{Sym}_{\mathcal{S}}$ of $K_{\mathcal{S}}$ where each colour is identified with a permutation of $\mathcal{S}$. The generating set of our partite presentation $P$ comprises the formal symbols

$$
\mathcal{U}=\left\{e, e^{-1}\right\} \cup\left\{m_{i, j} \mid m \in M, i, j \in\{-1,1\} \text { where } m^{2} \neq 1\right\} \text { and }
$$

$\mathcal{I}=\left\{m_{i, j} \mid m \in M, i, j \in\{-1,1\}\right.$ where $\left.m^{2}=1\right\}$. Set $\mathcal{S}^{\prime}=\mathcal{U} \cup \mathcal{I}$, the generators of $P$. We need to associate a permutation $\phi(s)$ of the vertex classes with each $s \in \mathcal{S}^{\prime}$, and we do so by

$$
\phi:{ }_{e, e^{-1}}^{m_{i}} \stackrel{m}{m}_{1 \mathcal{S}} .
$$

Let $\theta: \vec{E}\left(K_{\mathcal{S}}\right) \rightarrow M \cup M^{-1}$ be the colouring of $K_{\mathcal{S}}$ by $M \cup M^{-1}$. We can think of $\theta$ as a map from $\mathcal{S} \times \mathcal{S} \backslash\{(s, s) \mid s \in \mathcal{S}\}$ to $M \cup M^{-1}$ where $\theta(a, b)(a)=b$. Let $S=\left\{s, s^{-1} \mid s \in \mathcal{S}\right\}$ be $\mathcal{S}$ with formal inverses. Define a map $\chi: S \times S \backslash\left\{\left(s, s^{-1}\right) \mid s \in\right.$ $\mathcal{S}\} \rightarrow \mathcal{S}^{\prime}$ where

$$
\chi(a, b)= \begin{cases}e & \text { if } a=b \in \mathcal{S} \\ e^{-1} & \text { if } a=b \notin \mathcal{S} \\ m_{i, j} & \text { if } \theta(a, b)=m \text { where } a^{i}, b^{j} \in \mathcal{S} .\end{cases}
$$


Here we make the identification that $\left(m_{i, j}\right)^{-1}=\left(m^{-1}\right)_{-j,-i}$.

We now define the sets of relators $\mathcal{R}_{a}, a \in \mathcal{S}$ of $P$. For each relator $r:=a_{1} a_{2} \ldots a_{k} \in$ $\mathcal{R}$ we add

$\chi(r):=\chi\left(a_{1}, a_{2}\right) \chi\left(a_{2}, a_{3}\right) \ldots \chi\left(a_{k-1}, a_{k}\right) \chi\left(a_{k}, a_{1}\right)$ to $\mathcal{R}_{a_{1}^{ \pm 1}}$. (These are the relators of the first kind as explained at the beginning of the proof.) Lastly, we add relations (of the second kind) corresponding to the star of each vertex of $\Gamma$ as follows. Let $a_{1} \ldots a_{k} \in$ $W_{\mathcal{S}}$ be any word equalling the identity in $M F_{P}$, and add $\chi\left(a_{1}, a_{2}\right) \ldots \chi\left(a_{k}, a_{1}\right)$ to $\mathcal{R}_{a_{1}^{ \pm 1}}$, where $\chi\left(s, s^{-1}\right)$ is the empty word. Let $\mathcal{R}^{\prime}:=\left\{\mathcal{R}_{a}, a \in \mathcal{S}\right\}$. We have now constructed our presentation $P:=\left\langle\mathcal{S}|\mathcal{U}| \mathcal{I}|\phi| \mathcal{R}^{\prime}\right\rangle$.

Next, we prove that $\mathrm{PCay}(P)$ is isomorphic to $L(\Gamma)$. First label

$$
\begin{aligned}
& V(L(\Gamma))=\{[(g, g s)] \mid g \in G, s \in \mathcal{S}\} \text { and } \vec{E}(L(\Gamma)) \\
& =\left\{\left(g, s_{1}, s_{2}\right) \mid g \in G, s_{1}, s_{2} \in \mathcal{S} \cup \mathcal{S}^{-1}, s_{1} \neq s_{2}^{-1}\right\}
\end{aligned}
$$

so that the edge $\left(g, s_{1}, s_{2}\right)$ connects $\left[\left(g, g s_{1}\right)\right]$ and $\left[\left(g s_{1}, g s_{1} s_{2}\right)\right]$. Let $C:=C(P)$ be the presentation graph of $P$. Then we can define a map $\epsilon: L(\Gamma) \rightarrow C$ by letting $\epsilon([(g, g s)])=s$ and letting $\epsilon\left(\left(g, s_{1}, s_{2}\right)\right)$ be the edge of colour $\chi\left(s_{1}, s_{2}\right)$ coming from $s_{1}^{ \pm 1} \in \mathcal{S}$. One can show that the relations in $\mathcal{R}_{x}$ hold in $L(\Gamma)$ for all $x \in \mathcal{S}$. It remains to show that these relations suffice.

Intuitively we are going to argue that any closed walk $p$ in $L(\Gamma)$ is labelled by some $r \in\langle\langle\mathcal{R}\rangle\rangle_{M F_{P}}$ interwoven with relations coming from the stars at the vertices. One can observe this by just projecting $p$ to a closed walk in $\Gamma$, where after some cancelations happening within the stars of vertices, we are left with a closed walk labelled by a word $r$ than can be expressed in terms of the relators in $\mathcal{R}$. We proceed with this formally.

Define a topological map $\Phi: L(\Gamma) \rightarrow \Gamma$ by mapping $[(g, g s)] \in V(L(\Gamma))$ to the midpoint of the edge $(g, g s)$, and $\left(g, s_{1}, s_{2}\right) \in E(L(\Gamma))$ to the arc in the star of $g s_{1}$ connecting the midpoints of $\left[\left(g, g s_{1}\right)\right]$ and $\left[\left(g s_{1}, g s_{1} s_{2}\right)\right]$. Consider a closed walk $p$ in $L(\Gamma)$. We can write $p=\prod_{i=0}^{n-1}\left(g^{i}, s_{1}^{i}, s_{2}^{i}\right)$. As $\Phi(p)$ is a closed walk in $\Gamma$ we know it can be contracted to a path given by $g_{0} g_{1} \ldots g_{m-1}$ for $g_{i} \in G$. Now we want to group the edges of $p$ by the stars of vertices of $\Gamma$ they lie in. For this, we subdivide the interval $\{0, \ldots, n-1\}$ into disjoint subintervals $\left\{I_{j}\right\}_{j=0}^{m-1}$ such that $\Phi\left(g^{i}, s_{1}^{i}, s_{2}^{i}\right)$ lies in the star of $g_{j}$ for all $i \in I_{j}$ and $0 \leq j \leq m-1$ (we can assume without loss of generality that no $I_{j}$ has to be the union of an initial and a final subinterval of $\{0, \ldots, n-1\}$ by rotating $p$ appropriately). Thus $p=\prod_{j=0}^{m-1}\left(\prod_{i \in I_{j}}\left(g^{i}, s_{1}^{i}, s_{2}^{i}\right)\right)$.

To each $j$ we can also associate $s_{j} \in \mathcal{S} \cup \mathcal{S}^{-1}$ so that $g_{j} s_{j}=g_{j+1}$; these are the generators that $p$ uses in order to move from one star to the next.

We modify $p$ into a closed walk $p^{\prime}$ by inserting pairs of edges that have the same end-vertices and opposite directions each time that $p$ moves from one star to the next. More formally, we define

$$
p^{\prime}:=\prod_{j=0}^{m-1}\left(\left(\prod_{i \in I_{j}}\left(g^{i}, s_{1}^{i}, s_{2}^{i}\right)\right)\left(g_{j+1}, s_{j}^{-1}, s_{j-1}^{-1}\right)\left(g_{j-1}, s_{j-1}, s_{j}\right)\right) .
$$



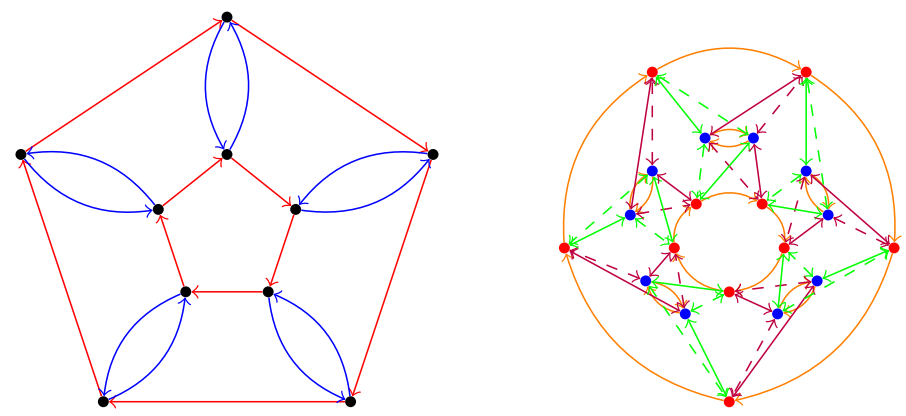

Fig. 7 Cay $\left\langle a, b \mid a^{5}, b^{2}, a b a^{-1} b^{-1}\right\rangle$ and its line graph

Notice that by contracting these pairs of opposite edges $\left(g_{j+1}, s_{j}^{-1}, s_{j-1}^{-1}\right)\left(g_{j-1}\right.$, $s_{j-1}, s_{j}$ ) we obtain $p$. Moreover, the sub-walk

$$
\left(\prod_{i \in I_{j}}\left(g^{i}, s_{1}^{i}, s_{2}^{i}\right)\right)\left(g_{j+1}, s_{j}^{-1}, s_{j-1}^{-1}\right)
$$

of $p^{\prime}$ stays within the star of $g_{j}$ by definition, and it is a closed walk starting and ending at $\left[\left(g_{j-1}, g_{j}\right)\right]$. Therefore, it is labelled by one of our relators of the second kind. Easily, $\Phi(p)$ is homotopic to $\Phi\left(p^{\prime}\right)$. Moreover,

$$
\begin{aligned}
\Phi\left(p^{\prime}\right) & =\prod_{j=0}^{m-1} \Phi\left(\left(\prod_{i \in I_{j}}\left(g^{i}, s_{1}^{i}, s_{2}^{i}\right)\right)\left(g_{j+1}, s_{j}^{-1}, s_{j-1}^{-1}\right)\right) \Phi\left(g_{j-1}, s_{j-1}, s_{j}\right) \\
& =\prod_{j=0}^{m-1} \Phi\left(g_{j-1}, s_{j-1}, s_{j}\right)
\end{aligned}
$$

since a closed walk contained in a star is 0-homotopic. Now $\prod_{j=0}^{m-1}\left(g_{j-1}, s_{j-1}, s_{j}\right)$ is a closed walk in $L(\Gamma)$ no three consecutive edges of which are contained in the star of a vertex of $\Gamma$ because of the way we chose the $I_{j}$. This implies that the word $s_{0} \ldots s_{m-1}$ labelling this walk is a relation of $\Gamma$, and so it can be written as a product of conjugates of relators $\mathcal{R}$. Recalling that each such relator was admitted as a relator (of the first kind) in $\mathcal{R}^{\prime}$, we conclude that the word labelling $p$ can be written as products of conjugates of words in $\mathcal{R}^{\prime}$.

We explicate an example of this below.

Example 6.2 Consider $D_{10}=\left\langle a, b \mid a^{5}, b^{2}, a b a^{-1} b^{-1}\right\rangle$, which has the Cayley graph and line graph thereof shown in Fig. 7. 

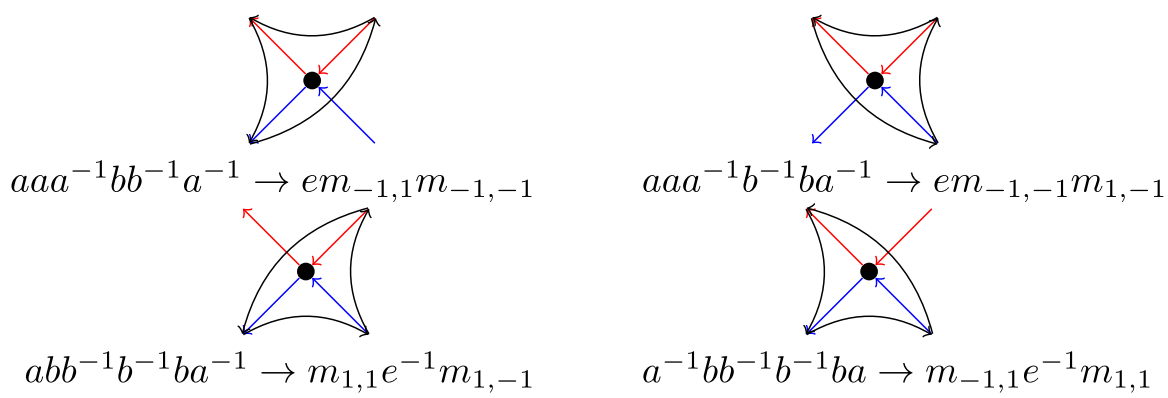

Fig. 8 Example of relations of the second kind

As $K_{\{a, b\}}$ is a single edge, we have $M=\{(1,2)\}=:\{m\}$ with $m_{i, j} \mapsto(1,2)$ for $i, j \in\{1,-1\}$ and $e, e^{-1} \mapsto(1)(2)$ as generators. Define the following function

$$
\begin{array}{rlrlrl}
a a & \rightarrow e & a b & \rightarrow m_{1,1} & b^{-1} a^{-1} & \rightarrow m_{-1,-1} \\
\chi: a^{-1} a^{-1} & \rightarrow e^{-1} & a b^{-1} & \rightarrow m_{1,-1} & b a^{-1} & \rightarrow m_{1,-1} \\
b b & \rightarrow e & a^{-1} b & \rightarrow m_{-1,1} & b^{-1} a & \rightarrow m_{-1,1} \\
b^{-1} b^{-1} & \rightarrow e^{-1} & a^{-1} b^{-1} & \rightarrow m_{-1,-1} & b a & \rightarrow m_{1,1} .
\end{array}
$$

The original relators $a^{5}, b^{2}, a b a^{-1} b^{-1}$ are thus translated into relators in the resulting partite presentation as follows: $a^{5} \rightarrow e^{5} \in \mathcal{R}_{a}, b^{2} \rightarrow e^{2} \in \mathcal{R}_{b}$ and $a b a^{-1} b^{-1} \rightarrow$ $m_{1,1} m_{1,-1} m_{-1,-1} m_{-1,1} \in \mathcal{R}_{a}$. Lastly, we add relations of the second kind shown in Fig. 8,

which are enough to generate the rest of the relations. The resulting partite presentation is

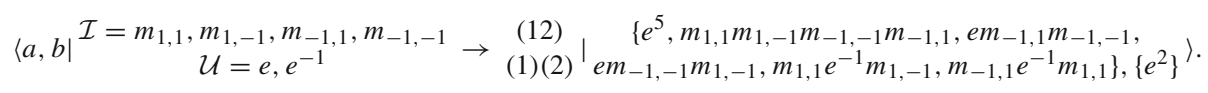

\section{Conclusion}

In this paper, we showed that every vertex-transitive graph admits a partite presentation, but we were not able to limit the number of vertex classes required. This suggests

Problem 7.1 Can every vertex-transitive graph on at least 3 vertices be represented as a partite Cayley graph so that each vertex class contains at least two vertices?

Define the Cayleyness of a (vertex-transitive) graph $\Gamma$ as the minimum number of vertex classes in any partite presentation of $\Gamma$. Thus $\Gamma$ is a Cayley graph if and only if it has Cayleyness 1.

Problem 7.2 Is there a vertex-transitive graph of Cayleyness (at least) $n$ for every $n \in \mathbb{N}$ ?

Since the Cayleyness of a vertex-transitive graph $\Gamma$ divides $|V(\Gamma)|$, a potential approach to answering this question is to enquire if for every prime $p \in \mathbb{N}$, there is a vertex-transitive graph on $p^{k}$ vertices for some $k \in \mathbb{N}$ that is not a Cayley graph. 
We observe that the Diestel-Leader graph $D L(m, n)$ for $m \neq n$ has infinite Cayleyness. This follows by combining Proposition 5.17 with the fact that these graphs are not quasi-isometric to any finitely generated group [7, Theorem 1.4]. This motivates

Problem 7.3 Does a locally finite vertex-transitive graph $\Gamma$ have finite Cayleyness if and only if $\Gamma$ is quasi-isometric to a Cayley graph?

We say that a locally finite (vertex-transitive) graph $\Gamma$ is finitely presented if it has a partite presentation with finitely many vertex classes and finitely many relators. Is this equivalent to $\pi_{1}(\Gamma)$ being generated by walks of bounded length? It would be interesting to generalise results about finitely presented groups such as [14] to finitely presented graphs in our sense.

It is not hard to show, using group presentations, that there are finitely many finite extensions of any finitely presented group. When it comes to vertex-transitive graphs the analogous question is still open and has been extensively studied, see [10,22,24] and references therein. We hope that partite presentations will be useful in developing an analogous proof.

Acknowledgements We thank Matthias Hamann for valuable discussions, and Derek Holt and Paul Martin for several corrections to an earlier draft of this work.

Open Access This article is licensed under a Creative Commons Attribution 4.0 International License, which permits use, sharing, adaptation, distribution and reproduction in any medium or format, as long as you give appropriate credit to the original author(s) and the source, provide a link to the Creative Commons licence, and indicate if changes were made. The images or other third party material in this article are included in the article's Creative Commons licence, unless indicated otherwise in a credit line to the material. If material is not included in the article's Creative Commons licence and your intended use is not permitted by statutory regulation or exceeds the permitted use, you will need to obtain permission directly from the copyright holder. To view a copy of this licence, visit http://creativecommons.org/licenses/by/4.0/.

\section{References}

1. Alspach, B.: The wonderful Walecki construction. Bull. Inst. Combin. Appl. 52, 7-20 (2008)

2. Baranyai, Z.: On the factorization of the complete uniform hypergraph. In Infinite and finite sets (Colloq., Keszthely, 1973; dedicated to P. Erdôs on his 60th birthday), Vol. I, volume 10, pages 91108.Vol. 10. Colloq. Math. Soc., (1975)

3. Csoka, E., Lippner, G.: Invariant random perfect matchings in Cayley graphs. Groups Geom. Dyn. 11(1), 211-243 (2017)

4. Dobson, E., Hujdurovic, A., Milanic, M., Verret, G.: Vertex-transitive CIS graphs. Europ. J. Combin. 44, 87-98 (2015)

5. Diestel, R.: Graph Theory. Graduate Texts in Mathematics, vol. 173, 3rd edn. Springer-Verlag, Berlin (2005)

6. Diestel, R., Leader, I.: A conjecture concerning a limit of non-Cayley graphs. J. Algebraic Combin. 14, 17-25 (2001)

7. Eskin, A., Fisher, D., Whyte, K.: Coarse differentiation of quasi-isometries I: spaces not quasi-isometric to Cayley graphs. Ann. Math. 176(1), 221-260 (2012)

8. Frucht, R., Graver, J.E., Watkins, M.E.: The groups of the generalized Petersen graphs. Proc. Cambridge Philos. Soc. 70, 211-218 (1971)

9. Gersten, S.M.: Intersections of finitely generated subgroups of free groups and resolutions of graphs. Invent. Math. 71(3), 567-591 (1983)

10. Gardiner, A.: and Praeger, C.E.: A geometrical approach to imprimitive graphs. Proc. Lond. Math. Soc. (3), 71(3):524-546, 1995 
11. Godsil, C., Royle, G.: Algebraic Graph Theory. Graduate Texts in Mathematics, vol. 207. Springer, Berlin (2001)

12. Hall, P.: On representatives of subsets. J. Lond. Math. Soc. 10, 26-30 (1935)

13. Hatcher, A.: Algebraic Topology. Cambridge University Press, Cambridge (2002)

14. Higman, G.: Subgroups of finitely presented groups. Proc. Roy. Soc. Lond. Ser. A Math. Phys. Sci. 262(1311), 455-475 (1961)

15. Kapovich, M.: A note on properly discontinuous actions. https://www.math.ucdavis.edu/ kapovich/ EPR/prop-disc.pdf

16. Leemann, P.H.: On subgroups and Schreier graphs of finitely generated groups. PhD Thesis. Université de Genéve, (2016)

17. Leighton, F.T.: On the decomposition of vertex-transitive graphs into multicycles. J. Res. Nat. Bur. Standards 88(6), 403-410 (1983)

18. Lovász, L.: Combinatorial structures and their applications: The factorization of graphs. Proceedings of the Calgary International Conference on Combinatorial Structures and their Applications held at the University of Calgary, Calgary, Alberta, Canada, vol. 1969. Science Publishers, New York-LondonParis, June. Gordon and Breach (1970)

19. Lauri, J., Scapellato, R.: Orbital Graphs and Strongly Regular Graphs. London Mathematical Society Lecture Note Series. Cambridge University Press, 2 edition, pp. 64-78 (2016)

20. Marušič, D.: On vertex symmetric digraphs. Discrete Math. 36(1), 69-81 (1981)

21. Milnor, J.: A note on curvature and fundamental group. J. Differential Geometry 2, 1-7 (1968)

22. Neganova, E.A., Trofimov, V.I.: Symmetric extensions of graphs. Izv. Ross. Akad. Nauk Ser. Mat. 78(4), 175-206 (2014)

23. Petersen, J.: Die Theorie der regulären Graphen. Acta Math. 15(1), 193-220 (1891)

24. Trofimov, V.I.: The finiteness of the number of symmetric extensions of a locally finite tree by means of a finite graph. Tr. Inst. Mat. Mekh. 21(3), 303-308 (2015)

25. Watkins, M.E.: Vertex-transitive graphs that are not Cayley graphs. In Cycles and rays (Montreal, PQ, 1987), volume 301 of NATO Adv. Sci. Inst. Ser. C Math. Phys. Sci., pages 243-256. Kluwer Acad. Publ., Dordrecht (1990)

26. Wendland, A.: Generalisations of groups and Cayley graphs. PhD Thesis. University of Warwick (2019)

Publisher's Note Springer Nature remains neutral with regard to jurisdictional claims in published maps and institutional affiliations. 